\title{
A Comparison of Game-Theoretic Models for Parallel Trade
}

\author{
Giorgio Gnecco - IMT School for Advanced Studies, Lucca, Italy \\ e-mail: giorgio.gnecco@imtlucca.it
}

Berna Tuncay - Koc University, Istanbul, Turkey

e-mail: betuncay@ku.edu.tr

Fabio Pammolli - Polytechnic University of Milan, Department of Management Engineering, and CADS, Human Technopole, Milan e-mail: fabio.pammolli@polimi.it

\begin{abstract}
Within the EU Single Market for medicines, differences in drug prices, regulations, and transaction costs may create, under suitable conditions, arbitrage opportunities well before patent expiration, giving an incentive to the occurrence of parallel trade. When this is permitted, parallel traders may obtain a profit from buying drugs in a country where prices are lower, then re-selling them in a country where prices are higher. This phenomenon may cause inefficiencies from a global welfare perspective, and reduce the manufacturers' incentive to invest in Research and Development (R \& D). Given this framework, in this paper, we investigate the efficiency (expressed in terms of the price of anarchy) of the subgame-perfect Nash equilibria associated with five dynamic noncooperative game-theoretic models for the parallel trade of pharmaceuticals. We also compare such models with regard to the manufacturer's incentive to invest in R \& D. More specifically, first we find in closed form the optimal value of the global welfare of two countries, which is obtained by solving a suitable quadratic optimization problem modeling the decision-making process of a global planner. Then, we use such a result to evaluate and compare the prices of anarchy of five games modeling the interaction between a manufacturer in the first country and a potential parallel trader in the second country. The first three games refer, respectively, to the cases of no parallel trade threat, parallel trade threat, and parallel trade occurrence at equilibrium. Then, we investigate two modifications of the third game, in which its transfer payment from the potential parallel trader to the manufacturer is, respectively, removed/determined by Nash bargaining. For completeness, we also consider a decision-theoretic model of no parallel trade threat. For what concerns the incentive for the manufacturer to invest in $\mathrm{R} \& \mathrm{D}$, the results of our numerical comparison show that the decision-theoretic model of no parallel trade threat is always the one with the highest incentive, whereas the two game-theoretic models of parallel trade threat/occurrence that do not include the transfer payment provide typically the smallest incentives. Moreover, the latter two models have the largest prices of anarchy (i.e., their equilibria have the smallest efficiencies). From a policy-making perspective, improvements are obtained if suitable countermeasures are taken to help the manufacturer recover from the costs of $\mathrm{R} \& \mathrm{D}$, such as the inclusion of a transfer payment in the model.
\end{abstract}

Keywords Economic applications of operations research · global welfare optimization · noncooperative game theory · subgame-perfect Nash equilibrium - numerical comparison of efficiency · incentive to invest in Research and Development

The authors acknowledge support from the Italian National Interest Project "Crisis Lab" (MIUR, PNR 2011-2013). G. G. is a member of GNAMPA-INdAM (Gruppo Nazionale per l'Analisi Matematica, la Probabilità e le loro Applicazioni - Istituto Nazionale di Alta Matematica). 
Drug prices, regulations, and transaction costs usually vary across countries. Under suitable conditions, differences in such factors may create an arbitrage opportunity well before patent expiration (i.e., before competition with generic drugs takes place), giving an incentive to the occurrence of a phenomenon known as parallel trade. When parallel trade is allowed by policymakers, parallel traders (e.g., distributors or agents trading with authorized distributors) may buy drugs in a country where prices are lower, then re-sell them in a country where prices are higher, and obtain a profit. As locally sourced and parallel traded drugs are produced by the same manufacturer, they are exactly the same (apart from different labeling/packaging), which creates opportunities for potential parallel traders. Parallel trade tends to increase prices in lower price countries, and to reduce them in higher price countries, decreasing also the revenues earned by manufacturers. This could make it difficult for the manufacturers to recover their past costs of Research and Development $(\mathrm{R} \& \mathrm{D})$, discouraging their future efforts in $\mathrm{R} \& \mathrm{D}$ of new drugs. It is also worth mentioning that negative effects of parallel trade on the manufacturers' incentive to do $\mathrm{R} \& \mathrm{D}$ and on other issues were already highlighted in several theoretical and empirical works (see, e.g., [9-11, 14,23, 28, 32, 45], and Section 2 for their discussion, and more generally for a literature review). This is particularly relevant, since endogenous sunk costs, such as those associated with $\mathrm{R} \& \mathrm{D}$, can improve the product quality, raising its demand [43].

Relevance of the topic for trade in the European Union. In order to grasp the practical importance of the topic, we recall that in Europe the phenomenon of parallel trade is made possible by free movement of goods [24], which is one of its fundamental principles, as being supported by European Union Treaty resolutions. As an example, parallel trade of pharmaceuticals in Europe is based on the concept of "community exhaustion" of intellectual property rights (IPRs) [47]. Once a patented drug has been sold in a country inside the European Union, the IPR holder has no longer the right to restrict selling that drug in other countries still inside the European Union (however, this does not exhaust such a restriction right outside the European Union). In Europe, free movement of goods is practically unlimited, with some exceptions in case of its negative influence on some selected markets. In particular, free trade in Europe is allowed for pharmaceutical products, whenever they are available on the market in at least one of its member states. As discussed in [9], parallel trade of pharmaceuticals within the European Union determines a conflict among different principles. On the one hand, countries generally differ in their health policies, which leads to differences in their prices, even when the marketed drug is identical. On the other hand, free movement of goods inside Europe makes it possible for parallel traders to obtain a profit from the possible arbitrage opportunity due to such price differences. In more quantitative terms, according to the recent study [23] made for the European Parliament, with reference to the main importing countries of the European Union, the market share of parallel-traded medicines has been reported between $1.7 \%$ (Finland) and $16.5 \%$ (Denmark). The phenomenon is particularly relevant since, according to the same study, medicines constitute the third largest cost component in the health care budgets of European countries, which spend more than 26 billion Euros per year on R \& D. However, as reviewed by the European Court of Justice, such an expenditure in $\mathrm{R} \& \mathrm{D}$ may not be incentivated by parallel trade.

Relevance of the topic for Operations Research. It is worth mentioning that the study of manufacturer's strategies to deal with potential competition from parallel trade has recently attracted interest also in Operations Research journals. Indeed, since parallel traders and manufacturers can be modeled as different agents with their own objectives, it is natural to investigate parallel trade using noncooperative game-theoretic models. For example, the paper [48] presents consumer rebates (i.e., payments from the manufacturer to the consumer, e.g., in the form of coupons [1,7,12]) as a possible tool to bypass competition from parallel trade, whereas [21] employs techniques from Operations Research to study the effect of investments in demand-enhancing activities to boost authorized channel demand as a mechanism to lower the pressure from parallel trade. Finally, [25] analyses the effects on pricing of centralization/decentralization of a multinational firm in the presence of competition from parallel traders.

Contributions of the work. Within this game-theoretic framework, in the paper we adopt the concept of price of anarchy [27] as a means to investigate the efficiency of equilibria to noncooperative game-theoretic models of parallel trade (and in one case, of the optimal solution to a related decision-theoretic model). Loosely speaking, for a 
noncooperative game, the price of anarchy measures the "worst-case" loss of efficiency of an equilibrium, which is due to the selfish behavior of its players. When the price of anarchy is "large", the rules of the game should be modified to achieve a much more efficient worst-case equilibrium. On the other hand, when it is "small", no change in the model is really needed. In the present context, the price of anarchy is the ratio between the optimal value of the global welfare (i.e., the one obtained by a hypothetical global planner, by solving a suitable optimization problem) and its value obtained in correspondence of the "worst" equilibrium of the game. A similar definition holds for a decision-theoretic model, with the equilibrium replaced by an optimal solution to the associated optimization problem.

In summary, the aim of the paper consists in comparing the effects of different levels of parallel trade freedom on the price of anarchy, considering various models (four from the literature, and two original modifications of an existing model). Our investigation takes also into account the total fixed cost of R \& D (and, more generally, the total fixed cost of market entry). This makes it possible to compare, for the various models, the decision by the manufacturer whether to invest or not to invest in R \& D. In order to compute the price of anarchy, we adopt the Bentham model for the global welfare function, formulating and solving in closed form suitable quadratic optimization problems modeling the decision-making process of the global planner (see Propositions 4.1 and 4.2), after expressing in closed form the surpluses of all the entities involved in the model. Then, we compare the expressions of the price of anarchy (and of the incentive for the manufacturer to invest in R \& D) obtained for three noncooperative games corresponding to different levels of parallel trade freedom: two dynamic noncooperative games proposed in [36] to model the interaction between a manufacturer and a distributor, which assume, respectively, no parallel trade threat from the distributor, and parallel trade threat from the distributor (but no occurrence of parallel trade at equilibrium), and a third dynamic noncooperative game proposed in [31], for which the manufacturer is able to take over the distributor's surplus through a transfer payment, and there is occurrence of parallel trade at equilibrium (see Propositions 5.2, 5.3, and 5.4). We also introduce and investigate two modifications of this third model. In the first modification, the transfer payment is removed. In the second modification, it is still present, but it is determined by Nash bargaining between the manufacturer and the distributor. In this way, the manufacturer is able to take over only part of the distributor's surplus. For completeness, we include in the comparison also a decision-theoretic model of no parallel trade threat from [34], in which the manufacturer is the only decision maker. For all the models, we find closed-form expressions for the price of anarchy, and we also prove that this is nondecreasing with respect to the total fixed cost of $\mathrm{R} \& \mathrm{D}$ when the global planner decides to invest in R \& D (see Proposition 6.2 (i), (ii)).

Our main findings in the successive numerical comparison are summarized as follows. We show that, in the ranges considered for the parameters, the game-theoretic models of parallel trade threat/occurrence that do not include the transfer payment provide the largest prices of anarchy, hence their equilibria have the smallest efficiencies. Instead, the decision-theoretic model of no parallel trade threat and the game-theoretic model of parallel trade occurrence with Nash bargaining have typically the smallest prices of anarchy. For what concerns the incentive for the manufacturer to invest in R \& D, we show numerically that the decision-theoretic model of no parallel trade threat is always the one with the largest incentive, whereas the two game-theoretic models of parallel trade threat/occurrence that do not include the transfer payment provide typically the smallest incentives. This is reasonable, since in those cases, the manufacturer is unable to take over (part of) the distributor's profit through the transfer payment. However, a larger incentive (still smaller than the one provided by the decision-theoretic model of no parallel trade threat) is obtained when one introduces, as a countermeasure, the transfer payment in the model. The results are in agreement with several studies (see, e.g., $[10,11,45]$ ), which highlight the negative effects of parallel trade, and the need to take suitable countermeasures to help the manufacturer recover from $\mathrm{R} \& \mathrm{D}$ costs.

Structure of the paper. The paper is organized as follows. Section 2 describes works in the literature related to parallel trade. Section 3 describes the adopted two-country model for the trade of pharmaceuticals. In Section 4 , we express in closed form the optimal value of the global welfare of the two countries, when parallel trade is permitted/forbidden. The two dynamic noncooperative games proposed in [36] to model no parallel trade threat/parallel trade threat are shortly summarized in Section 5, together with the third one proposed in [31] to model the occurrence of parallel trade at equilibrium. The section summarizes also a decision-theoretic model of no parallel trade threat from [34], 
and presents two original modifications of the game-theoretic model from [31]. Then, in Section 6, we evaluate the prices of anarchy associated with all the models above, and highlight their dependence on the relative market size of the two countries, the parallel trade cost per-unit, and the total fixed cost of R \& D. Section 7 presents numerical results. Finally, Section 8 discusses the results and mentions possible extensions of the methodology to other models for parallel trade investigated in the literature. All the proofs are reported in the appendix.

\section{Related Literature}

According to [33], the literature about the determinants of parallel trade can be divided into three streams. On one hand, [30,31] focus on the difficulties encountered by multinational companies in prohibiting sales outside the authorized distribution chains. One the other hand, $[6,16]$ focus on international price differences due to price regulation by national governments, which are typical, e.g., of the pharmaceutical industry. Finally, according to [30], a third determinant of parallel trade is the incentive to parallel traders of free-riding on investments in marketing.

In the literature, there is a strong debate about the consequences of parallel trade on global welfare, and about the opportunity or not of permitting parallel trade $[9,20,24,40]$. For instance, according to the analysis presented in [9], trade usually increases global welfare, by making consumers in higher price countries benefit from lower prices in other countries, when these lower prices are motivated by a more efficient or lower cost technology. Nevertheless, still according to [9], in the case of pharmaceuticals parallel trade may reduce global welfare, as an effect of a more intensive regulation. Moreover, the presence of parallel trade often lowers the incentive of the manufacturer for product innovation [28]. In [14], this has been confirmed empirically (referring to the case of the German market of oral anti-diabetics) through a structural approach, by comparing a counterfactual scenario with the status quo market. Additionally, according to [23], most of the revenues originated by parallel trade are accrued not to the consumers, but to the parallel traders themselves. Another negative aspect of parallel trade of pharmaceuticals has to do with its increase in the risk of drug shortage, for both higher and lower price countries ${ }^{1}$ [38]. In lower price countries, indeed, parallel trade may cause drug shortage, when one cannot foresee the extent of drug exports. In higher price countries, when imported drugs are sold at a lower price than locally sourced ones, sales of the same drugs produced by national suppliers may be discouraged, possibly causing drug shortage also in this case. Additionally, parallel trade may increase prices in lower price countries [32]. It is worth mentioning that, according to [22], parallel trade may even have a positive effect on global welfare, e.g., when it implies a re-allocation of consumption from individuals with relatively less drug needs to individuals with relatively more drug needs. Nevertheless, according to the same reference, parallel trade reduces global welfare when it implies, instead, a re-allocation of consumption from individuals with relatively more drug needs to individuals with relatively less drug needs, and also in case one market is not served as a consequence of parallel trade. More generally, the effect of parallel trade on global welfare is considered in [22] as ambiguous, in the sense that the positive/negative influence of parallel trade on global welfare depends on which aspects are taken into account in the model (e.g., the need for the manufacturer to recover from the R \& D costs). A two-country model of the effects of parallel trade on $\mathrm{R} \& \mathrm{D}$ is investigated in [2], where the dependence on the size of the two countries is also taken into account. In case the negative effects of parallel trade dominate, the following are some ways to still prevent its occurrence:

(i) a uniform pricing scheme could be adopted in all the countries potentially involved in parallel trade: in such a case, potential parallel traders would have no incentive to do parallel trade, due to the presence of positive parallel trade costs (associated, e.g., with transportation/repackaging). Although such a uniform pricing scheme has global welfare losses, there are some situations in which it has good global welfare properties [46]. An extreme case of uniform pricing is represented by the so-called "external reference pricing" scheme, which consists in setting the price of a specific drug (or its reimbursement from the government) to the smallest price of the same drug

\footnotetext{
1 It is worth mentioning that, when patents expire, competition from generics makes the price differences tend to vanish, eliminating the
} possible arbitrage opportunity for parallel trade, which is its main profit motivation for parallel importers. 
(or of a group of similar drugs) among countries otherwise potentially involved in parallel trade (hence, referring to the smallest "external" reference price);

(ii) parallel trade may simply be forbidden ${ }^{2}$ : however, this would require the intervention of policymakers. In such a case, if also external reference pricing does not occur, the so-called "differential pricing" or "price discrimination" scheme (in which possibly different prices are used in different countries) is considered in [10] as a good way to minimize global welfare losses, when such prices are set according to the so-called Ramsey pricing model [39]. For the pharmaceutical sector, price discrimination is considered in [11] as global welfare-superior to uniform pricing schemes, when both static and dynamic efficiency (related, respectively, to the optimal use of existing products, and to the optimal investment in $\mathrm{R} \& \mathrm{D}$ ) are taken into account, as price discrimination can increase the manufacturer's incentive to invest in $\mathrm{R} \& \mathrm{D}^{3}$. As an example, according to [45], price discrimination could be implemented in European Union:

(a) either by a Treaty change or a voluntary agreement to power centralization;

(b) or through the creation of different blocks of high-income/low-income countries, with parallel trade permitted only in the same block;

(c) or under discounts/voluntary contractual agreements implemented confidentially.

Parallel trade and its consequences on the global welfare of the involved countries are also investigated in the literature through various noncooperative game-theoretic models [8, 20,31,33,36]. For instance, according to the two-country model developed in [31], restricting parallel trade is always advantageous for the manufacturer, but it may either increase or decrease the global welfare of the two countries. However, a different model is used in [20], showing that parallel trade may even increase the profit of a pharmaceutical firm, depending on its bargaining power and on the relative market size of the exporting country with respect to the importing one. In [33], two dynamic noncooperative games are proposed to investigate the equilibrium behavior of a manufacturer located in a country and a distributor belonging to a second country, when parallel trade from the distributor is, respectively, permitted/forbidden. In the first case, it is shown that parallel trade actually does not even occur at equilibrium (i.e., the quantity of the reimported product from the parallel trader is zero), but the threat of potential parallel trade (or parallel trade freedom) influences the equilibrium behavior of both players, changing the equilibrium prices and quantities of the product sold by each of them. Instead, in the noncooperative game-theoretic model examined by [31], parallel trade actually occurs at equilibrium, when parallel trade is permitted. For other noncooperative games modeling parallel trade, we refer the reader to the monograph [36]. Finally, decision-theoretic models for parallel trade (in which there is only the manufacturer as the decision maker) are considered in [34].

\section{A Two-Country Model of Trade}

In this section, we summarize (and extend through the possible insertion of a total fixed cost of market entry, which can be interpreted, e.g., as the total fixed cost of R \& D) the model for the trade of pharmaceuticals considered in [31] and [36], involving two countries, which are characterized by different demand functions of one specific product possibly subject to parallel trade. The model includes both cases in which there is parallel trade freedom/banning, and applies also to parallel trade of other typologies of products. More precisely, the following is assumed in the model. The first country (named "country $A$ " in the following) is the one in which a drug is produced by a manufacturer with a marginal cost of production equal to 0 (e.g., because it is negligible, or because the most relevant costs are the ones of $\mathrm{R} \& \mathrm{D}$ ). Since the drug can be also sold in a second country (named "country $B$ " in the following), the country $A$ is

\footnotetext{
2 To this aim, various alternatives to "community exhaustion" of IPRs, mentioned in Section 1, exist [31]. For instance, according to "national exhaustion", IPRs of a product are exhausted inside a country after its first sale within that country, but parallel trade is still forbidden from abroad. According to "regional exhaustion", IPRs are exhausted inside a group of countries after the first sale within one of them (hence, allowing for parallel trade among such countries), but parallel trade is still not permitted from the outside.

3 It is worth remarking that, in the context of the paper, the piecewise-linearity of the demand functions considered later in formulas (1) and (2) precludes the application of the analysis made in [41], which provides conditions under which uniform pricing with linear demand functions is, instead, global welfare-superior.
} 
the exporting country, whereas the country $B$ is the importing country. However, when parallel trade from the country $B$ to the country $A$ is permitted, the country $A$ is also the re-importing country. The demand functions of the drug in the two countries $A$ and $B$ are modeled by the following piecewise-linear functions, respectively (with $p_{A}, p_{B} \geq 0$ ):

$$
\begin{aligned}
& q_{A}=\max \left\{0, \gamma a-b p_{A}\right\}, \\
& q_{B}=\max \left\{0, a-b p_{B}\right\},
\end{aligned}
$$

where $q_{A}$ (respectively, $q_{B}$ ) is the quantity of the drug that the consumers in the country $A$ (respectively, $B$ ) are willing to buy at the price $p_{A}$ (respectively, $p_{B}$ ), $a, b>0$ are two constants (the same for both countries), and $\gamma>1$ (relative market size) is another constant, which describes the heterogeneity of the countries $A$ and $B$ with respect to the market size. Indeed, in the limiting case of very small prices, one gets that the quantity $q_{A}$ bought in the country $A$ is nearly equal to $\gamma a$, and the quantity $q_{B}$ bought in the country $B$ is nearly equal to $a$ (in other words, $a$ is the intercept of the demand function in country $B$ ). In that case, $\gamma$ measures the relative market size of the country $A$ with respect to the country $B$, since $q_{A} \simeq \gamma q_{B}$. Finally, $-b$ is the slope of both demand functions. After its production, the drug can be

(i) sold by the manufacturer of the country $A$ to the consumers of the country $A$ in quantity $q_{A}^{M, C}$ at the retail price $p_{A}^{M, C}$

(ii) sold by the manufacturer of the country $A$ to the distributor of the country $B$ in quantity $q_{B}^{M, D}$ at the wholesale price $p_{B}^{M, D}$

(iii) sold by the distributor of the country $B$ to the consumers of the country $B$ in quantity $q_{B}^{D, C}$ at the retail price $p_{B}^{D, C}$

(iv) (only when there is parallel trade freedom) sold by the distributor of the country $B$ to the consumers of the country $A$ in quantity $q_{A}^{D, C}$ at the retail price $p_{A}^{D, C}$. In doing this, the distributor incurs a fixed parallel trade cost per-unit $t \geq 0$. When, instead, parallel trade is forbidden, one sets $q_{A}^{D, C}=0$.

In this model, the distributor plays the role of potential parallel trader (in country $A$ only). The model of trade considered in [31], actually, slightly differs from the one in [36] for the simplifying assumptions $a=1$ and $b=1$ (which we do not make in the paper, redoing all the computations from [31] needed in our analysis for generic positive values of $a$ and $b$ ), and for the additional presence of a transfer payment $T_{P} \geq 0$ (franchise fee), which is paid by the distributor to the manufacturer, and which we also include in the model of this paper. Moreover, we also include in the model a total fixed cost $C_{F} \geq 0$ of market entry for the manufacturer, which is taken into account in the paper to establish whether it is advantageous to enter the market (then, producing the drug), or not to enter the market (then, producing nothing).

One can notice that, according to the model above, only the distributor can sell to the consumers in the country $B$. When there is parallel trade freedom, the distributor can also sell to the consumers in the country $A$ (in the model, parallel trade from the consumers in the country $B$ to the consumers in the country $A$ is always forbidden). Of course, one has also the constraint $q_{B}^{M, D}=q_{A}^{D, C}+q_{B}^{D, C}$, i.e., the total quantity of the product sold to the distributor (by the manufacturer) is equal to the total quantity of the product sold by the distributor (to the consumers in the countries $A$ and $B$ ). This simplifies to $q_{B}^{M, D}=q_{B}^{D, C}$ when parallel trade is forbidden. Finally, in this model $q_{A}=q_{A}^{M, C}+q_{A}^{D, C}$ is the quantity of the product sold to the consumers in the country $A$, and $q_{B}=q_{B}^{D, C}$ is the quantity of the product sold to the consumers in the country $B$. The whole process above is illustrated in Figure 1.

\section{Optimization of the Global Welfare Function through Quadratic Programming}

In this section, using the two-country model for the trade of pharmaceuticals described in Section 3, we first determine the surpluses of the manufacturer, of the distributor, and of the consumers in both countries. Then, on the basis of the obtained formulas, we provide expressions for the global welfare function under a specific and well-known model 


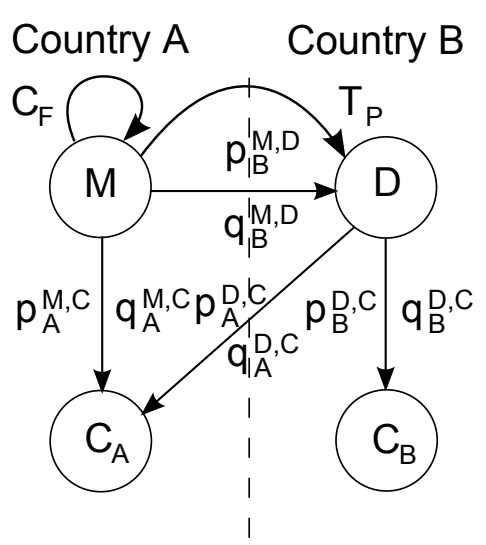

Fig. 1: The model of trade among the manufacturer, the distributor, and the consumers in the countries $A$ and $B$ when there is parallel trade freedom. When parallel trade is forbidden, one sets $q_{A}^{D, C}=0$.

for it (the Bentham model). Finally, we find in closed form the optimal value of the global welfare function itself (i.e., the one that could be found by the global planner, by solving a suitable optimization problem). Then, the results of this analysis are exploited in Section 6 as one of the tools needed to compute the price of anarchy for some models of parallel trade, which are presented in Section 5 .

To start the analysis, we need the following definition of surplus, for each of the entities involved.

Definition 4.1 In the present context:

- the manufacturer's surplus when selling a given quantity of a good is the difference between the total amount of money received from selling the good and its total fixed cost of market entry, when incurred ${ }^{4}$;

- the distributor's surplus is the total amount of money received from trading;

- for each country, the consumers' surplus is the definite integral (from 0 to the total quantity actually bought by the consumers) of the difference between the maximum price per-unit they would pay for any quantity of the good between 0 and the total quantity actually bought ${ }^{5}$, and the price per-unit of the good.

For the two-country model of trade of pharmaceuticals described in Section 3, it follows from Definition 4.1 that one has the following expressions for the surpluses of the manufacturer, of the distributor, and of the consumers in the countries $A$ and $B$.

(a) When the manufacturer enters the market:

(i) Manufacturer's surplus $S_{M}$ :

$$
S_{M}=p_{A}^{M, C} q_{A}^{M, C}+p_{B}^{M, D} q_{B}^{M, D}-C_{F}+T_{P}
$$

(ii) Distributor's surplus $S_{D}$ :

$$
S_{D}=\left(p_{A}^{D, C}-p_{B}^{M, D}-t\right) q_{A}^{D, C}+\left(p_{B}^{D, C}-p_{B}^{M, D}\right) q_{B}^{D, C}-T_{P} .
$$

(iii) Consumers' surplus $S_{C_{A}}$ in the country $A$ :

$$
S_{C_{A}}=\frac{\gamma a}{b}\left(q_{A}^{M, C}+q_{A}^{D, C}\right)-p_{A}^{M, C} q_{A}^{M, C}-p_{A}^{D, C} q_{A}^{D, C}-\frac{\left(q_{A}^{M, C}+q_{A}^{D, C}\right)^{2}}{2 b}
$$

(iv) Consumers' surplus $S_{C_{B}}$ in the country $B$ :

$$
S_{C_{B}}=\left(\frac{a}{b}-p_{B}^{D, C}\right) q_{B}^{D, C}-\frac{\left(q_{B}^{D, C}\right)^{2}}{2 b} .
$$

\footnotetext{
4 This specific definition takes into account the total fixed cost of market entry, since in the present setting the manufacturer could even decide not to enter the market, hence not to incur the total fixed cost of market entry.

5 This maximum price depends on the quantity, and is provided by the inverse demand function.
} 
(b) When the manufacturer does not enter the market:

$$
S_{M}=S_{D}=S_{C_{A}}=S_{C_{B}}=0
$$

The proof of formula (5), which is longer than the ones of formulas (3) and (4), (6), and (7), is reported in the appendix.

We now consider the most usual functional form for the global welfare function $G W$, which is the Bentham model. In the following, we refer to such model using the superscript " $(B)$ ". It is defined as the sum of all the surpluses. We use the subscript "PTF" to denote the situation in which there is parallel trade freedom, whereas the subscript "PTB" refers to the case in which parallel trade is banned. When the manufacturer enters the market and parallel trade is permitted, the Bentham global welfare function has the following expression $G W_{P T F}^{(B)}$, which is a function only of the total fixed cost of market entry and of the traded quantities (for simplicity, here we have removed the dependence on $q_{B}^{M, D}$, using the constraint $\left.q_{B}^{M, D}=q_{A}^{D, C}+q_{B}^{D, C}\right)^{6}$ :

$$
\begin{aligned}
& G W_{P T F}^{(B)}\left(C_{F}, q_{A}^{M, C}, q_{A}^{D, C}, q_{B}^{D, C}\right) \\
= & S_{M}+S_{D}+S_{C_{A}}+S_{C_{B}} \\
= & \frac{\gamma a}{b}\left(q_{A}^{M, C}+q_{A}^{D, C}\right)-\frac{\left(q_{A}^{M, C}+q_{A}^{D, C}\right)^{2}}{2 b}-t q_{A}^{D, C}+\frac{a}{b} q_{B}^{D, C}-\frac{\left(q_{B}^{D, C}\right)^{2}}{2 b}-C_{F} .
\end{aligned}
$$

When the manufacturer enters the market and parallel trade is not permitted, it has the following simpler expression $G W_{P T B}^{(B)}$ :

$$
\begin{aligned}
G W_{P T B}^{(B)}\left(C_{F}, q_{A}^{M, C}, q_{B}^{D, C}\right) & =S_{M}+S_{D}+S_{C_{A}}+S_{C_{B}} \\
& =\frac{\gamma a}{b} q_{A}^{M, C}-\frac{\left(q_{A}^{M, C}\right)^{2}}{2 b}+\frac{a}{b} q_{B}^{D, C}-\frac{\left(q_{B}^{D, C}\right)^{2}}{2 b}-C_{F}
\end{aligned}
$$

which is obtained from (8) by setting $q_{A}^{D, C}=0$. Finally, when the manufacturer does not enter the market, one has $G W_{P T B}^{(B)}=0$.

In the following, we find the optimal value of the Bentham global welfare function for a global planner who maximizes it under suitable assumptions.

Remark 4.1 In the next analysis, we consider two situations: one is when the manufacturer enters the market, and the other one is when the manufacturer does not enter the market. Of the two situations, of course, the global planner prefers the one with the largest value of the global welfare (or is indifferent, when they produce the same value). Since the second situation is associated with a zero value of the global welfare, the optimal global welfare for the global planner is always non-negative. Moreover, an inspection of the proofs of the next Propositions 4.1 and 4.2 shows that, for $C_{F}=0$, the corresponding optimal solution for the global planner is always compatible with non-negative surpluses for all the entities involved (manufacturer, distributor, and consumers of both countries). When $C_{F}>0$, in case the global planner suggests the manufacturer to enter the market (because this is preferable for the global planner) and side payments to the manufacturer are inserted in the trade model, the optimal global welfare can still be implemented by prices such that all the surpluses are non-negative, the surplus of the manufacturer being larger than or equal to the zero surplus that it would obtain in case of no market entry. Finally, such proofs show also that every time the optimal value of the global welfare for the global planner is zero, this implies that it is not possible for the manufacturer to have a positive surplus, even under a suboptimal allocation for the global planner. This implies that the maximum value of $C_{F}$ for which the manufacturer chooses to enter the market under any pricing scheme ${ }^{7}$ is always smaller than or equal to the maximum value of $C_{F}$ under which the global planner suggests the manufacturer to enter the market.

\footnotetext{
${ }^{6}$ Formula (8) is derived using the expressions for $S_{M}, S_{D}, S_{C_{A}}$, and $S_{C_{B}}$ provided by formulas (3), (4), (5), and (6), respectively, taking into account the constraint $q_{B}^{M, D}=q_{A}^{D, C}+q_{B}^{D, C}$, and observing that all the terms containing prices cancel out in the summation.

7 E. g., one of the pricing schemes considered in Section 5.
} 
We first consider the case in which the manufacturer enters the market and parallel trade is permitted. Then, in order to find the optimal value of the Bentham global welfare $G W_{P T F}^{(B)}$ provided by formula (8), the global planner has to solve the following optimization problem, which is a quadratic programming problem:

$$
\begin{array}{cl}
\underset{q_{A}^{M, C}, q_{A}^{D, C}, q_{B}^{D, C}}{\operatorname{maximize}} & G W_{P T F}^{(B)}\left(C_{F}, q_{A}^{M, C}, q_{A}^{D, C}, q_{B}^{D, C}\right) \\
\text { s.t. } & q_{A}^{M, C}, q_{A}^{D, C}, q_{B}^{D, C} \geq 0 .
\end{array}
$$

Of course, when the manufacturer does not enter the market and parallel trade is permitted, there is actually nothing to optimize, and the optimal value of $G W_{P T F}^{(B)}$ is 0 . The following proposition refers to both cases.

Proposition 4.1 When the manufacturer enters the market: the optimal value of the objective $G W_{P T F}^{(B)}$ of the optimization problem (10) modeling parallel trade freedom under the Bentham global welfare model is

$$
\left(G W_{P T F}^{(B)}\right)^{\circ}=\frac{\left(\gamma^{2}+1\right) a^{2}}{2 b}-C_{F} .
$$

When the manufacturer does not enter the market: the optimal value of $G W_{P T F}^{(B)}$ is

$$
\left(G W_{P T F}^{(B)}\right)^{\circ}=0
$$

For the case in which parallel trade is forbidden, one has to use the expression (9) for the Bentham global welfare function $G W_{P T B}^{(B)}$, and solve the following optimization problem, which is still a quadratic programming problem:

$$
\begin{gathered}
\underset{q_{A}^{M, C}, q_{B}^{D, C}}{\operatorname{maximize}} G W_{P T B}^{(B)}\left(C_{F}, q_{A}^{M, C}, q_{B}^{D, C}\right) \\
\text { s.t. } \quad q_{A}^{M, C}, q_{B}^{D, C} \geq 0 .
\end{gathered}
$$

Again, when the manufacturer does not enter the market and parallel trade is forbidden, there is actually nothing to optimize, and the optimal value of $G W_{P T B}^{(B)}$ is 0 . The following proposition refers to both cases.

Proposition 4.2 When the manufacturer enters the market: the optimal value of the objective $G W_{P T B}^{(B)}$ of the optimization problem (13) modeling parallel trade banning under the Bentham global welfare model is

$$
\left(G W_{P T B}^{(B)}\right)^{\circ}=\frac{\left(\gamma^{2}+1\right) a^{2}}{2 b}-C_{F} .
$$

When the manufacturer does not enter the market: the optimal value of $G W_{P T B}^{(B)}$ is

$$
\left(G W_{P T B}^{(B)}\right)^{\circ}=0
$$

Hence, Propositions 4.1 and 4.2 provide the same optimal values for the Bentham global welfare function for the two cases of parallel trade freedom/banning, respectively.

\section{A Decision Theoretic Model, and Five Game-Theoretic Models for Parallel Trade}

In practice, the prices and quantities of the model presented in Section 3 in general cannot be chosen realistically by a global planner, because - given the demand functions - they depend on the interaction between the manufacturer and the distributor, and on their choices. In the following, we summarize the results of the analyses of the noncooperative game-theoretic models considered in [31] and [36] to represent the interaction between the manufacturer and the distributor, assuming that the manufacturer enters the market, hence incurs the total fixed cost $C_{F} \geq 0$ of market entry (see Remark 5.6 at the end of the section for the extension to the case in which the manufacturer can decide not to enter the market). For completeness, we also consider a decision-theoretic model with no parallel trade threat and 
no distributor, which was presented in [34]. The optimal solution of this decision-theoretic model determines prices and quantities at optimality that are in general different from the ones determined by the global planner. Similar comments hold for the subgame-perfect Nash equilibria of the other noncooperative game-theoretic models.

Remark 5.1 We recall that a subgame-perfect Nash equilibrium of a dynamic noncooperative game is an equilibrium such that its players' strategies constitute a Nash equilibrium for every subgame of the original dynamic noncooperative game. Not all Nash equilibria are also subgame-perfect Nash equilibria. The difference between a generic Nash equilibrium and a subgame-perfect Nash equilibrium is that the latter requires an additional assumption on the players' behavior, which is called sequential rationality. Basically, this means that if, at some decisional stage, one player changes its action with respect to the one suggested by its equilibrium strategy at that stage, then the other player can detect and adapt to that change at its successive decisional stage. Hence, the concept of subgame-perfect Nash equilibrium takes into account some form of feedback in the players' behavior, which makes it a realistic refinement of the concept of Nash equilibrium. We refer, e.g., to [17] for more details on the previous definitions, and also on the other game-theoretic concepts mentioned in this section.

The following models are ordered according to an increasing level of complexity in the interaction between the manufacturer and the distributor (apart from the first model, in which there is actually no distributor).

(i) A decision-theoretic model (no parallel trade threat and no distributor) [34, Section 4.1]. In this first model, the distributor is absent, and there is only the manufacturer as the unique decision maker, who is able to sell directly not only to the country $A$, but also to the country $B$. Hence, in this case, $p_{B}^{D, C}$ and $q_{B}^{D, C}$ of the original trade model presented in Section 3 are replaced by $p_{B}^{M, C}$ and $q_{B}^{M, C}$. The optimal prices in the two countries (and the associated quantities sold to the respective consumers) are obtained by maximizing the manufacturer's surplus. Equivalently, such a model can be obtained by assuming that the distributor of the trade model presented in Section 3 coincides with (or is owned by) the manufacturer. In this case, due to the parallel trade cost per-unit, there would be no advantage in doing parallel trade. So, this is a model of no parallel trade threat, likewise the next one (in which, however, the manufacturer and the distributor have different objectives). In the following, we refer to the present model using the superscript " $N o P T T\left(N_{o D}\right)$ " to recall that, in this case, there is no parallel trade threat, and no distributor (independent from the manufacturer) is present.

Proposition 5.1 At the unique optimal solution of the manufacturer's surplus maximization problem modeling no parallel trade threat and no distributor, the surpluses of the manufacturer and of the consumers in the two countries have the following expressions:

$$
\left\{\begin{array}{l}
S_{M}^{\circ, N o P T T(N o D)}=\frac{\gamma a}{2 b} \frac{\gamma a}{2}+\frac{a}{2 b} \frac{a}{2}-C_{F}=\frac{\left(\gamma^{2}+1\right) a^{2}}{4 b}-C_{F}, \\
S_{C_{A}^{\circ}, N o P T T(N o D)}^{\circ}=\frac{\gamma a}{b} \frac{\gamma a}{2}-\frac{\gamma a}{2 b} \frac{\gamma a}{2}-\frac{\left(\frac{\gamma a}{2}\right)^{2}}{2 b}=\frac{\gamma^{2} a^{2}}{8 b}, \\
S_{C_{B}, N o P T T(N o D)}^{\circ}=\frac{a}{b} \frac{a}{2}-\frac{a}{2 b} \frac{a}{2}-\frac{\left(\frac{a}{2}\right)^{2}}{2 b}=\frac{a^{2}}{8 b} .
\end{array}\right.
$$

(ii) First noncooperative game-theoretic model: parallel trade is forbidden (no parallel trade threat in the presence of a distributor), i.e., $q_{A}^{D, C}=0$ [36]. The interaction of the players (here, the manufacturer and the distributor) is described by a dynamic noncooperative game with perfect and complete information, and two stages. In the first stage, the manufacturer sets the wholesale price $p_{B}^{M, D}$ for the distributor. Then, in the second stage, the distributor sets the retail price $p_{B}^{D, C}$ for the consumers in the country $B$. No transfer payment is paid by the distributor to the manufacturer. The game is solved in [35] by backward induction (the final result is also reported in [36]). In the following, we refer to this model using the superscript "NoPTT $(D)$ " to recall that, in this case, there is no parallel threat, and a distributor (independent from the manufacturer) is present. 
Proposition 5.2 At any subgame-perfect Nash equilibrium of the game modeling no parallel trade threat in the presence of the distributor, the surpluses of the manufacturer, of the distributor, and of the consumers in the two countries have the following expressions:

$$
\left\{\begin{array}{l}
S_{M}^{\text {(s.p.Nash,NoPTT(D)) }}=\frac{\gamma a}{2 b} \frac{\gamma a}{2}+\frac{a}{2 b} \frac{a}{4}=\frac{\left(2 \gamma^{2}+1\right) a^{2}}{8 b}-C_{F}, \\
S_{D}^{\text {(s.p.Nash,NoPTT(D)) }}=\left(\frac{3 a}{4 b}-\frac{a}{2 b}\right) \frac{a}{4}=\frac{a^{2}}{16 b}, \\
S_{C_{A}}^{\text {(s.p.Nash,NoPTT(D)) }}=\frac{\gamma a}{b} \frac{\gamma a}{2}-\frac{\gamma a}{2 b} \frac{\gamma a}{2}-\frac{\left(\frac{\gamma a}{2}\right)^{2}}{2 b}=\frac{\gamma^{2} a^{2}}{8 b}, \\
S_{C_{B}}^{(\text {s.p.Nash,NoPTT(D)) }}=\left(\frac{a}{b}-\frac{3 a}{4 b}\right) \frac{a}{4}-\frac{\left(\frac{a}{4}\right)^{2}}{2 b}=\frac{a^{2}}{32 b} .
\end{array}\right.
$$

(iii) Second noncooperative game-theoretic model: parallel trade is permitted, but no parallel trade occurs at equilibrium (parallel trade threat) [36]. Again, the interaction of the players (the manufacturer and the distributor) is described by a dynamic noncooperative game with perfect and complete information, and three stages. In the first stage, the manufacturer sets the wholesale price $p_{B}^{M, D}$ for the distributor. Then, in the second stage, the distributor sets the retail price $p_{B}^{D, C}$ for the consumers in the country $B$. In the third stage, the manufacturer and the distributor choose simultaneously the prices $p_{A}^{M, C}$ and $p_{A}^{D, C}$ at which they sell the product to the consumers in the country $A$, according to a Bertrand duopoly model [17]. No transfer payment is paid by the distributor to the manufacturer. Again, the game is solved in [35] by backward induction. The result of the equilibrium analysis (also reported in [36]) depends on the value of the parallel trade cost per-unit $t$. More precisely, two thresholds for $t$ are defined in [36]:

$$
\begin{aligned}
t_{l} & = \begin{cases}\frac{a}{2 b}\left(\gamma-\frac{5}{2}\right) & \text { if } \gamma \geq \frac{5}{2} \\
0 & \text { otherwise, }\end{cases} \\
t_{h} & =\frac{a}{2 b}(\gamma-1) .
\end{aligned}
$$

Of course, $t_{l} \leq t_{h}$. Hence, one distinguishes among low values for $t\left(0 \leq t<t_{l}\right)$, intermediate values for $t$ $\left(t_{l} \leq t \leq t_{h}\right)$, and high values for $t\left(t \geq t_{h}\right)$. Notice that the first case is meaningful only when $t_{l}>0$. It is also worth noting that the threshold $t_{h}$ represents the boundary between a credible and a not-credible parallel trade threat to the manufacturer. Likewise in [36], in the following we use the symbols $l, i$, and $h$ to denote the three respective cases. Moreover, we use the superscript "PTT" to recall that the present game-theoretic model refers to parallel trade threat.

Proposition 5.3 At any subgame-perfect Nash equilibrium of the game modeling parallel trade threat, the surpluses of the manufacturer, of the distributor, and of the consumers in the two countries have the following expressions:

$$
\left\{\begin{aligned}
S_{M}^{(\text {s.p.Nash }, l, P T T)} & =\left(\frac{a}{6 b}(2 \gamma+1)+\frac{t}{3}\right)\left(\frac{a}{6}(4 \gamma-1)-\frac{b t}{3}\right)-C_{F}, \\
S_{D}^{(\text {s.p.Nash }, l, P T T)} & =0, \\
S_{C_{A}}^{\text {(s.p.Nash }, l, P T T)} & =\frac{\gamma a}{b}\left(\frac{a}{6}(4 \gamma-1)-\frac{b t}{3}\right)-\left(\frac{a}{6 b}(2 \gamma+1)+\frac{t}{3}\right)\left(\frac{a}{6}(4 \gamma-1)-\frac{b t}{3}\right) \\
& -\frac{\left(\frac{a}{6}(4 \gamma-1)-\frac{b t}{3}\right)^{2}}{2 b}, \\
S_{C_{B}}^{(\text {s.p.Nash }, l, P T T)} & =0,
\end{aligned}\right.
$$




$$
\begin{aligned}
& \left\{\begin{aligned}
S_{M}^{(\text {s.p.Nash }, i, P T T)}= & \left(\frac{a}{6 b}(2 \gamma+1)+\frac{t}{3}\right)\left(\frac{a}{6}(4 \gamma-1)-\frac{b t}{3}\right) \\
& +\left(\frac{a}{6 b}(2 \gamma+1)-\frac{2 t}{3}\right)\left(\frac{a}{12}(5-2 \gamma)+\frac{b t}{3}\right)-C_{F}, \\
S_{D}^{\text {s.p.Nash }, i, P T T)}= & \left(\frac{a}{12 b}(2 \gamma+7)-\frac{t}{3}-\left(\frac{a}{6 b}(2 \gamma+1)-\frac{2 t}{3}\right)\right)\left(\frac{a}{12}(5-2 \gamma)+\frac{b t}{3}\right), \\
S_{C_{A}}^{(\text {s.p.Nash }, i, P T T)}= & -\left(\frac{a}{6 b}(2 \gamma+1)+\frac{t}{3}\right)\left(\frac{a}{6}(4 \gamma-1)-\frac{b t}{3}\right)+\frac{\gamma a}{b}\left(\frac{a}{6}(4 \gamma-1)-\frac{b t}{3}\right) \\
& -\frac{\left(\frac{a}{6}(4 \gamma-1)-\frac{b t}{3}\right)^{2}}{2 b}, \\
S_{C_{B}}^{(\mathrm{s.p.Nash}, i, P T T)=} & -\left(\frac{a}{12 b}(2 \gamma+7)-\frac{t}{3}\right)\left(\frac{a}{12}(5-2 \gamma)+\frac{b t}{3}\right)+\frac{a}{b}\left(\frac{a}{12}(5-2 \gamma)+\frac{b t}{3}\right) \\
& -\frac{\left(\frac{a}{12}(5-2 \gamma)+\frac{b t}{3}\right)^{2}}{2 b},
\end{aligned}\right. \\
& \left\{\begin{array}{l}
S_{M}^{\text {(s.p.Nash, } h, P T T)}=\frac{\gamma a}{2 b} \frac{\gamma a}{2}+\frac{a}{2 b} \frac{a}{4}=\frac{\left(2 \gamma^{2}+1\right) a^{2}}{8 b}-C_{F} \\
S_{D}^{\text {(s.p.Nash, } h, P T T)}=\left(\frac{3 a}{4 b}-\frac{a}{2 b}\right) \frac{a}{4}=\frac{a^{2}}{16 b}, \\
S_{C_{A}}^{\text {(s.p.Nash } h, P T T)}=\frac{\gamma a}{b} \frac{\gamma a}{2}-\frac{\gamma a}{2 b} \frac{\gamma a}{2}-\frac{\left(\frac{\gamma a}{2}\right)^{2}}{2 b}=\frac{\gamma^{2} a^{2}}{8 b}, \\
S_{C_{B}}^{\text {(s.p.Nash, }, P T T)}=\left(\frac{a}{b}-\frac{3 a}{4 b}\right) \frac{a}{4}-\frac{\left(\frac{a}{4}\right)^{2}}{2 b}=\frac{a^{2}}{32 b} .
\end{array}\right.
\end{aligned}
$$

One can notice that, for each of these subgame-perfect Nash equilibria, parallel trade actually does not occur at equilibrium. However, parallel trade freedom has an influence on the equilibrium behavior of the players (as one can see by comparing such equilibria with the ones obtained when parallel trade is forbidden; see the first game-theoretic model reported in this section).

(iv) Third noncooperative game-theoretic model: parallel trade is permitted, and it occurs at equilibrium (parallel trade occurrence (with transfer payment)) [31]. Also in this case, the interaction of the players (the manufacturer and the distributor) is described by a dynamic noncooperative game with perfect and complete information, and two stages. In the first stage, the manufacturer sets the wholesale price $p_{B}^{M, D}$ for the distributor, together with a transfer payment $T_{P} \geq 0$, chosen inside the set of transfer payments that guarantee a non-negative surplus for the distributor (such a subset is determined as a by-product of the analysis performed in [31] of the equilibrium behaviors in the later stages). Then, in the second stage, the manufacturer and the distributor decide simultaneously the quantities $q_{A}^{M, C}$ and $q_{A}^{D, C}$ to be sold, respectively, to the consumers in the country $A$. At the same time, the distributor also decides the quantity $q_{B}^{D, C}$ to be sold to the consumers in the country $B$. The price $p_{A}^{M, C}=p_{A}^{D, C}$ is determined by a Cournot duopoly model [17], whereas the price $p_{B}^{D, C}$ is obtained by equating the offer from the distributor and the demand of the consumers in the country $B$. Also this game is solved in [31] by backward induction. Again, the result of the equilibrium analysis depends on the value of the parallel trade cost per-unit $t$. The following result refers to choices of the parameters for which there is actual occurrence of parallel trade at the (unique) subgame-perfect Nash equilibrium. In this case, the manufacturer is able to extract the whole profit obtained by the distributor through the transfer payment. Here, we use the superscript "PTO" to recall that the present game-theoretic model refers to the parallel trade occurrence at equilibrium. 
Proposition 5.4 Let $1<\gamma \leq 2$ and $0 \leq t<\frac{3 \gamma a}{14 b}$. At the unique subgame-perfect Nash equilibrium of the game modeling parallel trade occurrence with transfer payment, the surpluses of the manufacturer, of the distributor, and of the consumers in the two countries have the following expressions:

$$
\left\{\begin{aligned}
S_{M}^{\text {s.p.Nash,PTO) }=} & \frac{\left(\gamma a+\frac{2 \gamma a+8 b t}{13}+b t\right)^{2}}{9 b}+\frac{2 \gamma a+8 b t}{13 b} \frac{\gamma a-2 \frac{2 \gamma a+8 b t}{13}-2 b t}{3} \\
& +\frac{\left(\gamma a-2 \frac{2 \gamma a+8 b t}{13}-2 b t\right)^{2}}{9 b}+\frac{a^{2}-\left(\frac{2 \gamma a+8 b t}{13}\right)^{2}}{4 b}-C_{F} \\
S_{D}^{\text {(s.p.Nash,PTO) }}= & 0, \\
S_{C_{A}}^{\text {(s.p.Nash,PTO) }=} & \frac{\left(\gamma a-\frac{5 \gamma a+7 b t}{13}\right)^{2}}{2 b}, \\
S_{C_{B}}^{\text {(s.p.Nash,PTO) }}= & \frac{\left(\frac{a}{2}-\frac{\gamma a+4 b t}{13}\right)^{2}}{2 b} .
\end{aligned}\right.
$$

Remark 5.2 The main difference between the second noncooperative game-theoretic model and the third one is the additional presence, in the latter, of the transfer payment $T_{P}$. Another difference is in the use of a different duopoly model to analyze the competition between the manufacturer and the distributor in the country $A$. An inspection of the proof of [31, Proposition 1] shows that one reason for the choice $1<\gamma \leq 2$ in the third noncooperative gametheoretic model is the desire to obtain an equilibrium for which the country $B$ is served (see Remark 5.3 for additional technical details about this issue). In spite of the differences in the two models, this is in good agreement with the results of the analysis of the second noncooperative game-theoretic model, for which the country $B$ is also served at equilibrium for $1<\gamma \leq 2$. Indeed, in that model, the importing country is not served at equilibrium only for $0 \leq t \leq t_{l}$. However, this condition is never satisfied for $1<\gamma \leq 2$, since in that case one gets $t_{l}=0$ from formula (18). Finally, the condition $0 \leq t<\frac{3 \gamma a}{14 b}$ in the third noncooperative game-theoretic model is required to have parallel trade at equilibrium (see [31, Proposition 2]).

Remark 5.3 We provide in the following some insights about the reason for which, when $1<\gamma \leq 2$, the country $B$ is served at equilibrium in the third noncooperative game-theoretic model. An inspection of the proof of [31, Proposition 1] shows that, for $1<\gamma \leq 2$, there are two possibilities at the equilibrium in the second stage (before the equilibrium values of $p_{B}^{M, D}$ and $T_{P}$ are determined in the first stage): either $p_{B}^{M, D}$ is smaller than or equal to the threshold $\frac{\gamma a-2 b t}{2 b}$, or it is larger than that threshold. In the first case, one can see from the proof of [31, Proposition 1] that the country $B$ is served. In the second case, one can see from that proof that there is no parallel trade occurrence, and that the manufacturer is able to maximize its profit in the country $A$ as if the competition from the distributor in that country were not present. Among the possible choices for the price $p_{B}^{M, D}$ above the threshold $\frac{\gamma a-2 b t}{2 b}$, there is at least one (typically, an interval of values for that price) for which also the country $B$ is served: since the manufacturer has already obtained the maximum possible profit from the country $A$, and is also able to obtain additional profit by selling to the distributor and extracting from the distributor (via the transfer payment $T_{P}$ ) its whole profit coming from serving the country $B$, the manufacturer has the incentive to choose such a price. Concluding, for $1<\gamma \leq 2$, when the manufacturer optimizes $p_{B}^{M, D}$ and $T_{P}$ in the first stage, the resulting optimal solution is such that the country $B$ is served.

Summarizing, the optimal solution of the decision-theoretic model and the equilibria of the three noncooperative game-theoretic models above represent, respectively, the following situations:

(a) a case in which the manufacturer sets different prices in the two countries, irrespectively of the parallel trade cost per-unit (since there is no independent distributor);

(b) a case in which the manufacturer still sets different prices in the two countries, irrespectively of the parallel trade cost per-unit, but taking into account the behavior of the distributor (since parallel trade is forbidden, but the market in the importing country is served by the distributor, who is independent from the manufacturer); 
(c) a case in which, depending on the parallel trade cost per-unit $t$, the manufacturer either sells nothing in the country $B$ (clearly preventing the occurrence of parallel trade), or sets a price difference equal to $t$ (discouraging potential parallel traders), or acts as in the case (b) (when parallel trade does not really represent a threat to the manufacturer). When $t=0$, the second subcase models a particular uniform pricing scheme;

(d) a case in which the manufacturer accepts the possible presence of parallel trade, and reacts to this by decreasing its prices in both countries with respect to the situation described in the case (b) (since, for its admissible values of the parameters, one has $\frac{5 \gamma a+7 b t}{13 b}<\frac{\gamma a}{2 b}$ and $\left.\frac{2 \gamma a+8 b t}{13 b}<\frac{a}{2 b}\right)$, whereas the distributor reacts by setting a retail price in the country $B$ larger than the wholesale price set by the manufacturer in the same country for the case (b) (since $\left.\frac{a}{2 b}+\frac{\gamma a+4 b t}{13 b}>\frac{a}{2 b}\right)$.

In the following, we also introduce and analyze two modifications ${ }^{8}$ of the third noncooperative game-theoretic model, by changing its assumptions about the transfer payment $T_{P}$. In the first modification, we constrain $T_{P}$ to be equal to 0 (i.e., there is no transfer payment, making the resulting model more similar to the second noncooperative game-theoretic model). This is done to see the effect of the presence of the transfer payment in the original model. In the second modification, we assume instead that there is still a transfer payment $T_{P}$, but that it is determined by Nash bargaining between the manufacturer and the distributor, together with the wholesale price $p_{B}^{M, D}$ for the distributor. This assumption is more realistic than the corresponding one made in the original model, for which the transfer payment is chosen at the equilibrium in such a way to extract from the distributor its whole profit, transferring it to the manufacturer.

(v) First modification of the third noncooperative game-theoretic model (no transfer payment in the first stage). In this case, the difference with respect to the third noncooperative game-theoretic model is that, in the first stage, the transfer payment is set to $T_{P}=0$. Hence, in that stage, the manufacturer optimizes its profit with respect to the wholesale price $p_{B}^{M, D}$ for the distributor. The following Proposition 5.5 summarizes the results of our equilibrium analysis. We use the superscript " $N o T_{P}$ " to recall that the present game-theoretic model is the modification of the third model obtained by removing the transfer payment. To reduce the number of cases considered in the analysis (still investigating two significant cases), we have made slightly different assumptions on the admissible values for the parameters with respect to the ones imposed for the third noncooperative gametheoretic model (other choices for the parameters lead, in any case, to a similar analysis). In particular, we have chosen the parameters in such a way that the country $B$ is served at equilibrium (see the proof, reported in the appendix, for details about the prices and quantities at equilibrium). In one case addressed in the analysis (small parallel trade cost per-unit $t$ ), there is still parallel trade occurrence at equilibrium, likewise for the original model. In the other case (large parallel trade cost per-unit $t$ ), there is no parallel trade occurrence at equilibrium. Likewise for the original model, we have not considered the case of a very large value for $t$, since in that case parallel trade is never profitable for the distributor, so its occurrence at equilibrium can be excluded from the very beginning of the analysis.

Proposition 5.5 (i) Let $1<\gamma \leq 2$ and $0 \leq t<\frac{9(\gamma-1) a}{30 b}$. At the unique subgame-perfect Nash equilibrium of the first modification of the third noncooperative game, the surpluses of the manufacturer, of the distributor, and of the consumers in the two countries have the following expressions:

\footnotetext{
8 The authors acknowledge an anonymous reviewer for suggesting the development of these two modifications during the first review
} round of the manuscript. 


$$
\left\{\begin{aligned}
& S_{M}^{\left(\text {s.p.Nash, } N o T_{P}\right)}= \frac{\gamma a+\frac{(10 \gamma+9) a-8 b t}{38 b} b+b t}{3 b} \frac{\gamma a+\frac{(10 \gamma+9) a-8 b t}{38 b} b+b t}{3} \\
&+\frac{(10 \gamma+9) a-8 b t}{38 b}\left(\frac{\gamma a-2 \frac{(10 \gamma+9) a-8 b t}{38 b} b-2 b t}{3}+\frac{a-\frac{(10 \gamma+9) a-8 b t}{38 b} b}{2}\right)-C_{F} \\
& S_{D}^{\left(\text {s.p.Nash, } N o T_{P}\right)}=\left(\frac{\gamma a+\frac{(10 \gamma+9) a-8 b t}{38 b} b+b t}{3 b}-\frac{(10 \gamma+9) a-8 b t}{38 b}-t\right) \frac{\gamma a-2 \frac{(10 \gamma+9) a-8 b t}{38 b} b-2 b t}{3} \\
&+\left(\frac{a+\frac{(10 \gamma+9) a-8 b t}{38 b} b}{2 b}-\frac{(10 \gamma+9) a-8 b t}{38 b}\right) \frac{a-\frac{(10 \gamma+9) a-8 b t}{38 b} b}{2}, \\
& S_{C_{A}}^{\left(\text {s.p.Nash, } N o T_{P}\right)}= \frac{\gamma a}{b}\left(\frac{\gamma a+\frac{(10 \gamma+9) a-8 b t}{38 b} b+b t}{3}+\frac{\gamma a-2 \frac{(10 \gamma+9) a-8 b t}{38 b} b-2 b t}{3}\right) \\
&-\frac{\gamma a+\frac{(10 \gamma+9) a-8 b t}{38 b} b+b t}{3 b} \frac{\gamma a+\frac{(10 \gamma+9) a-8 b t}{38 b} b+b t}{3} \\
&-\frac{\gamma a+\frac{(10 \gamma+9) a-8 b t}{38 b} b+b t}{3 b} \frac{\gamma a-2 \frac{(10 \gamma+9) a-8 b t}{38 b} b-2 b t}{3} \\
&-\frac{\left(\frac{\gamma a+\frac{(10 \gamma+9) a-8 b t}{38 b} b+b t}{3}+\frac{\gamma a-2 \frac{(10 \gamma+9) a-8 b t}{38 b} b-2 b t}{3}\right)^{2}}{2 b}, \\
& S_{C_{B}}^{(\text {s.p.Nash, NoTP })=}\left.\left(\frac{a}{b}-\frac{a+\frac{(10 \gamma+9) a-8 b t}{38 b} b}{2 b}\right) \frac{a-\frac{(10 \gamma+9) a-8 b t}{38 b} b}{2}-\frac{a-\frac{(10 \gamma+9) a-8 b t}{38 b}}{2}\right)^{2} \\
& 2 b
\end{aligned}\right.
$$

(ii) Let $1<\gamma \leq 2$ and $\frac{9(\gamma-1) a}{30 b} \leq t \leq \frac{(\gamma-1) a}{2 b}$. At the unique subgame-perfect Nash equilibrium of the first modification of the third noncooperative game, the surpluses of the manufacturer, of the distributor, and of the consumers in the two countries have the following expressions:

$$
\left\{\begin{array}{rl}
S_{M}^{\left(\text {s.p.Nash }, N o T_{P}\right)} & =\frac{\gamma a}{2 b} \frac{\gamma a}{2}+\frac{\gamma a-2 b t}{2 b} \frac{(2-\gamma) a+2 b t}{4}-C_{F} \\
& =\frac{\gamma^{2} a^{2}}{4 b}+\frac{\gamma a-2 b t}{2 b} \frac{(2-\gamma) a+2 b t}{4}-C_{F}, \\
S_{D}^{\left(\text {s.p.Nash }, N o T_{P}\right)} & =\left(\frac{(2+\gamma) a-2 b t}{4 b}-\frac{\gamma a-2 b t}{2 b}\right) \frac{(2-\gamma) a+2 b t}{4}, \\
S_{C_{A}}^{\left(\text {s.p.Nash }, N o T_{P}\right)} & =\frac{\gamma a}{b} \frac{\gamma a}{2}-\frac{\gamma a}{2 b} \frac{\gamma a}{2}-\frac{\left(\frac{\gamma a}{2}\right)^{2}}{2 b}=\frac{\gamma^{2} a^{2}}{8 b}, \\
S_{C_{B}}^{\left(\text {s.p.Nash }, N o T_{P}\right)} & =\left(\frac{a}{b}-\frac{(2+\gamma) a-2 b t}{4 b}\right) \frac{(2-\gamma) a+2 b t}{4}-\frac{\left(\frac{(2-\gamma) a+2 b t}{4}\right)^{2}}{2 b}
\end{array} .\right.
$$

Remark 5.4 It is worth mentioning that, since the proof of [31, Proposition 1] (on which the first steps in the proof of Proposition 5.5 are based) shows that having the country $B$ not served is not profitable for the manufacturer for $1<\gamma \leq 2$ (see also Remark 5.3), this case has not been considered in our analysis of the first modification of the third game-theoretic model ${ }^{9}$, reported in the proof of Proposition 5.5.

(vi) Second modification of the third noncooperative game-theoretic model (Nash bargaining in the first stage). In this case, the difference with respect to the third noncooperative game-theoretic model is that, in the first stage, the transfer payment $T_{P}$ is determined by Nash bargaining ${ }^{10}$ between the manufacturer and the distributor, together with the wholesale price $p_{B}^{M, D}$ for the distributor. The following Proposition 5.6 summarizes the results of our equilibrium analysis. In this case, parallel trade occurs at equilibrium, and the country $B$ is served (again, see the proof, reported in the appendix, for details about the prices, quantities, and transfer payment at equilibrium). We use the superscript "NashB" to recall that the present game-theoretic model is the modification of the third model obtained by including Nash bargaining in the first stage.

\footnotetext{
${ }^{9}$ However, it has been considered in the analysis of its second modification, to determine the disagreement point in the Nash bargaining problem modeling the first stage of the game (see the proof of Proposition 5.6).

10 See, e.g., [37, Section 2.4] and the Appendix for details about Nash bargaining.
} 
Proposition 5.6 Let $1<\gamma \leq 2$ and $0 \leq t<\frac{3 \gamma a}{14 b}$. At the unique subgame-perfect Nash equilibrium ${ }^{11}$ of the second modification of the third noncooperative game, the surpluses of the manufacturer, of the distributor, and of the consumers in the two countries have the following expressions:

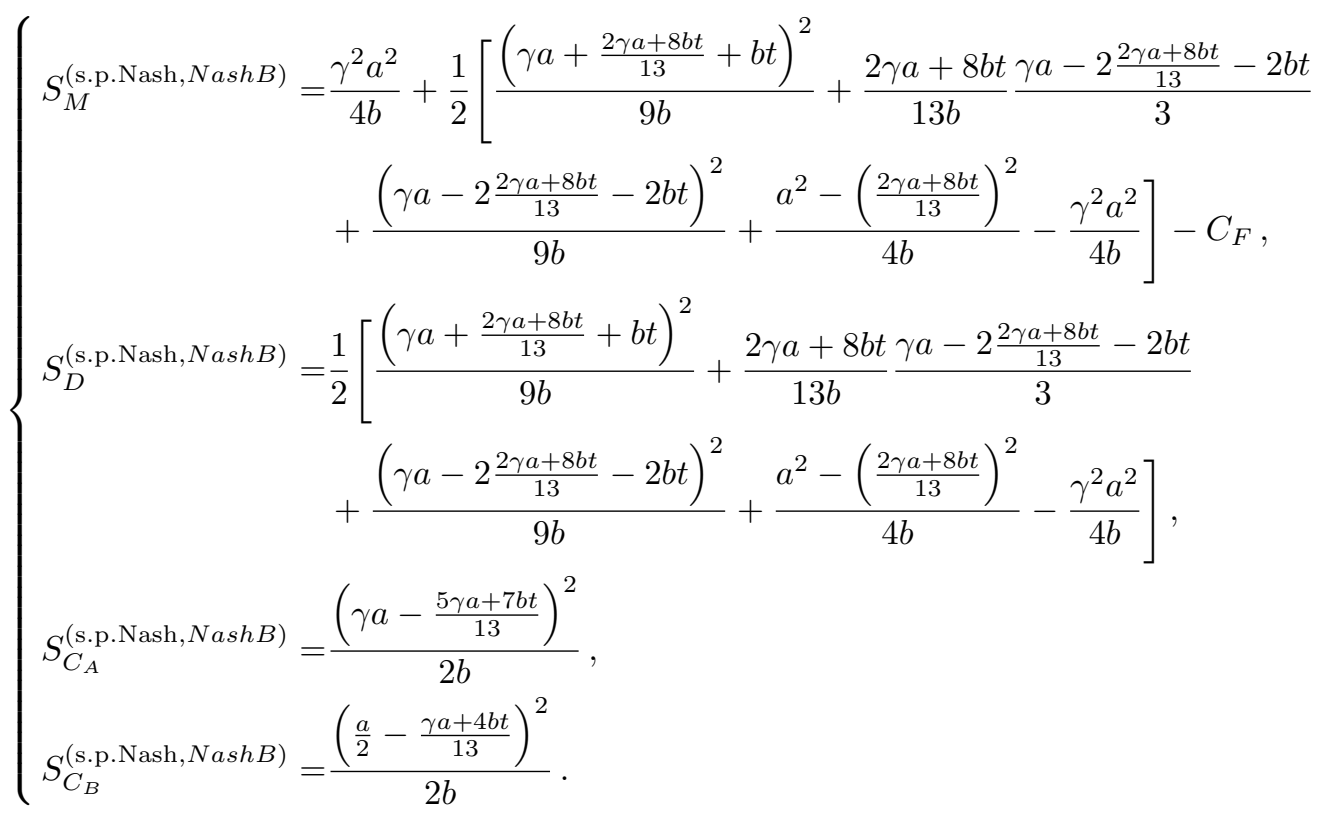

Remark 5.5 It is worth noting that, in all the models in which parallel trade is allowed, the manufacturer can always set a price large enough in country $B$ such as to prevent parallel trade in that country, without necessarily changing the price in country $A$. Hence, even in the worst case for the profit-maximizing manufacturer, its optimal surplus is always larger than or equal to the portion of its optimal surplus coming from the country $A$ when parallel trade is not allowed.

Remark 5.6 All the equilibria of the game-theoretic models above refer to the case in which the manufacturer decides to enter the market, hence, it incurs the total fixed cost $C_{F}$. In addition, one can see straightforwardly that all such equilibria are associated with non-negative surpluses of the distributor and of the consumers in the two countries, whereas the surplus of the manufacturer can be negative if the total fixed cost $C_{F}$ is too large (but it is always nonnegative for $C_{F}=0$ ). However, due to their subgame-perfectness, the equilibria above change only slightly if one adds to the previous game-theoretic models the constraint that the surplus of the manufacturer has to be non-negative, making the manufacturer decide, in an additional preliminary decisional stage, not to enter the market when its surplus is negative in case of market entry. By adding such a preliminary stage, indeed, one obtains exactly the same expressions of the prices and quantities at equilibrium when the manufacturer decides to enter the market and the associated surplus of the manufacturer is non-negative, whereas all the quantities and surpluses become 0 otherwise. Finally, with this modification the values of the global welfare associated with such equilibria are always non-negative. Similar comments hold for the optimal solution of the decision-theoretic model above.

Remark 5.7 It is worth remarking that the first noncooperative game-theoretic model of parallel trade threat and the first modification of the third game-theoretic model with no transfer payment in the first stage can be considered, in a sense, as "pure" models of parallel trade, in which the distributor simply buys from the country $B$ and sells back to the country $A$, without paying the transfer payment to the manufacturer. Instead, the third game-theoretic model and its second modification including Nash bargaining in the first stage can be considered as models of parallel trade that include an additional "corrective action" (the inclusion of the transfer payment), which allows the manufacturer to take over (part of) the surplus of the distributor, with possible benefits for the manufacturer and for the global welfare (see Section 7 for a numerical comparison, whose results confirm these expectations).

\footnotetext{
11 Here, for uniformity of notation with respect to the other game-theoretic models, we still use the term "subgame-perfect Nash equilibrium", since it is well-known that Nash bargaining can be interpreted as a limit case of the Rubinstein bargaining solution (see, e.g., [18, Section 15.5]), which is the subgame-perfect Nash equilibrium to a suitable noncooperative game.
} 
Remark 5.8 For each of the six models above, one can easily find the maximum value of $C_{F}$ for which the manufacturer has the incentive to enter the market. This is equal to the corresponding expression of the manufacturer's surplus obtained for $C_{F}=0$. For instance, for the game modeling no parallel trade threat in the presence of the distributor, such a maximum value is equal to $\frac{\left(2 \gamma^{2}+1\right) a^{2}}{8 b}$, due to Proposition 5.2. In accordance to the end of Remark 4.1, this value is smaller than $\frac{\left(\gamma^{2}+1\right) a^{2}}{2 b}$, which is the maximum value of $C_{F}$ (derived from Proposition 4.2 ) for which the global planner suggests the manufacturer to enter the market.

\section{An Application of the Price of Anarchy to the Six Models for Parallel Trade}

In this section, for each of the six models described in Section 5, we apply an adaptation of the concept of price of anarchy [27] to the context of the paper, in order to compute the loss of efficiency in the optimization of the global welfare function, which is incurred when moving from the optimal solution determined by the global planner to the prices and quantities obtained at the "worst" optimal solution/subgame-perfect Nash equilibrium ${ }^{12}$ of the model.

The following definition formalizes our adaptation of the definition of price of anarchy ${ }^{13}$ (PoA) from [27] to the six models above. In the following definitions, in order to have non-negative values for the global welfare function in all the ratios, we assume that both the global planner and the manufacturer choose their strategies according to Remarks 4.1 and 5.6, respectively, i.e., taking into account even the possibility not to enter the market.

Definition 6.1 For each of the six models described in Section 5, the price of anarchy (PoA) is defined as the ratio whose numerator is the optimal value of the global welfare obtained by the global planner under the same conditions of parallel trade banning/freedom, and the denominator is the value of the global welfare associated with the "worst" optimal solution/subgame-perfect Nash equilibrium of the model (i.e., the one associated with the smallest value of the global welfare).

Remark 6.1 Definition 6.1 differs from the one given in [27] for the two following reasons, which are needed to adapt the original definition of price of anarchy to the context of the paper.

(i) In Definition 6.1, the numerator refers to the optimal value determined by the global planner, whereas the denominator refers to the value associated with the worst optimal solution/equilibrium, whereas [27] does the opposite in its definition. This change in the definition is due to the fact that here we are considering the maximization of the global welfare, whereas [27] refers to a cost minimization problem. With this modification, one obtains in the present context a value of the price of anarchy that is always ${ }^{14}$ greater than or equal to 1 , likewise in the definition given in [27] for cost minimization problems.

(ii) In the context of the paper, we consider optimal solutions/subgame-perfect Nash equilibria, instead than simply Nash equilibria.

The importance of the concept of price of anarchy (in both Definition 6.1 and in its original version stated in [27]) derives from the observation that it allows one to compare the outcomes of different models, detecting when a change in each model (due, e.g., to the possible intervention of a policymaker, such as a government) is needed to have a much more efficient (worst-case) optimal solution/equilibrium.

Remark 6.2 For each of the six models described in Section 5, one could also consider a "normalized" price of anarchy. This can be defined as the ratio between the maximum of the optimal values of the global welfare obtained by the global planner under each of the two conditions of parallel trade banning/freedom, and the value of the global welfare associated with the worst optimal solution/subgame-perfect Nash equilibrium of the model. However, in the present context, this additional concept is not needed, since, for each of the six models, the price of anarchy and the normalized

\footnotetext{
12 Optimal solution in the case of the first model, subgame-perfect Nash equilibrium for the other five models.

13 Originally proposed in [26] under the name of "coordination ratio"; nowadays, the term "price of anarchy" is more common [27].

14 When the ratio in the definition of the price of anarchy has the indeterminate form $\frac{0}{0}$, by convention (and uniformity with respect to the other non-indeterminate cases), we assign to it the value 1 , as the numerator and the denominator are equal.
} 
price of anarchy coincide, due to Propositions 4.1 and 4.2. Indeed, the optimal value of the global welfare when parallel trade is forbidden is the same as its optimal value when it is permitted. Incidentally, for all the models considered in the paper, this makes equal the numerators in the expressions of the respective prices of anarchy.

Remark 6.3 For each fixed choice of one among the six models considered in the paper, of the parameters $a, b$, of the heterogeneity parameter $\gamma$, and of the parallel trade cost per-unit $t$, all the optimal solutions/subgame-perfect Nash equilibria have actually the same value of the global welfare, so there is actually no need for searching for the "worst" optimal solution/equilibrium, as all of them are equivalent in efficiency. This may not be true for other models of parallel trade banning/freedom.

In the following, we express the price of anarchy for the various models considered in the paper. In order to simplify the presentation, the following proposition provides expressions for the prices of anarchy associated with the Bentham global welfare model and the six models of parallel trade banning/freedom considered in Section 5 when $C_{F}=0$, a case for which both the global planner and the manufacturer prefer entering the market. In this situation, both the numerator and the denominator in the definition of the price of anarchy have simplified forms with respect to the general case. An interesting consequence of the next Proposition 6.1 is that, when the total fixed cost $C_{F}$ of market entry is equal to 0, the price of anarchy is a constant for the first model, it is a function only of the relative market size $\gamma$ for the second model, whereas, for the other four models, it is a function only of the relative market size $\gamma$ and of $t /\left(\frac{a}{b}\right)$, which is the ratio whose numerator is the parallel trade cost-per unit $t$, and the denominator is the ratio between the two parameters $a$ and $b$ of the demand function of the smallest country. Indeed, also the various thresholds on $t$ appearing in the other four models can be equivalently expressed as thresholds on $t /\left(\frac{a}{b}\right)$.

Proposition 6.1 (i) For the decision-theoretic model with no parallel trade threat and no distributor, the price of anarchy under the Bentham global welfare model has the following expression for $C_{F}=0$ :

$$
P o A_{N o P T T(N o D)}^{(B)}=\frac{\frac{\left(\gamma^{2}+1\right) a^{2}}{2 b}}{\frac{3\left(\gamma^{2}+1\right) a^{2}}{8 b}}=\frac{4}{3} .
$$

(ii) For the game modeling no parallel trade threat in the presence of the distributor, the price of anarchy under the Bentham global welfare model has the following expression for $C_{F}=0$ :

$$
\operatorname{PoA}_{N o P T T(D)}^{(B)}(\gamma)=\frac{\frac{\left(\gamma^{2}+1\right) a^{2}}{2 b}}{\frac{a^{2}}{32 b}\left(12 \gamma^{2}+7\right)}=\frac{16\left(\gamma^{2}+1\right)}{12 \gamma^{2}+7}
$$

(iii) For the game modeling parallel trade threat, the price of anarchy under the Bentham global welfare model has the following expression for $C_{F}=0$ :

$$
\operatorname{PoA}_{P T T}^{(B)}(\gamma, t)= \begin{cases}\frac{\frac{\left(\gamma^{2}+1\right) a^{2}}{2 b}}{\frac{a^{2}}{72 b}\left(32 \gamma^{2}-4 \gamma-1\right)-\frac{1}{18}\left(b t^{2}+a t+2 \gamma a t\right)} & \text { if } 0 \leq t<t_{l}, \\ =\frac{36\left(\gamma^{2}+1\right)}{32 \gamma^{2}-4 \gamma-1-4\left(t /\left(\frac{a}{b}\right)\right)^{2}-4(2 \gamma+1)\left(t /\left(\frac{a}{b}\right)\right)} & \frac{\left(\gamma^{2}+1\right) a^{2}}{2 b} \\ \frac{\frac{a^{2}}{288 b}\left(124 \gamma^{2}-44 \gamma+91\right)-\frac{1}{36}\left(4 b t^{2}+2 \gamma a t-5 a t\right)}{144\left(\gamma^{2}+1\right)} & \text { if } t_{l} \leq t \leq t_{h}, \\ \frac{\frac{\left(\gamma^{2}+1\right) a^{2}}{2 b}}{\frac{a^{2}}{32 b}\left(12 \gamma^{2}+7\right)} & \text { if } t>t_{h} . \\ =\frac{16\left(\gamma^{2}+1\right)}{12 \gamma^{2}+7} & \end{cases}
$$


(iv) For the game modeling parallel trade occurrence with transfer payment, the price of anarchy under the Bentham global welfare model has the following expression for $C_{F}=0,1<\gamma \leq 2$, and $0 \leq t<\frac{3 \gamma a}{14 b}$ :

$$
\begin{aligned}
\operatorname{PoA}_{P T O}^{(B)}(\gamma, t) & =\frac{\frac{\left(\gamma^{2}+1\right) a^{2}}{2 b}}{\frac{\frac{11}{26}(\gamma a)^{2}-\frac{6}{13}(\gamma a)(b t)+\frac{23}{26}(b t)^{2}+\frac{3}{8} a^{2}-\frac{1}{26}(\gamma a) a-\frac{2}{13} a(b t)}{b}} \\
& =\frac{\gamma^{2}+1}{\frac{11}{13} \gamma^{2}-\frac{12 \gamma+4}{13}\left(t /\left(\frac{a}{b}\right)\right)+\frac{23}{13}\left(t /\left(\frac{a}{b}\right)\right)^{2}+\frac{3}{4}-\frac{1}{13} \gamma}
\end{aligned}
$$

(v) For the first modification of the third game with no transfer payment in the first stage, the price of anarchy under the Bentham global welfare model has the following expression for $C_{F}=0,1<\gamma \leq 2$, and $0 \leq t<\frac{9(\gamma-1) a}{30 b}$ :

$$
\begin{aligned}
\operatorname{PoA}_{N o T_{P}}^{(B)}(\gamma, t) & =\frac{\frac{\left(\gamma^{2}+1\right) a^{2}}{2 b}}{\frac{4652 a^{2} \gamma^{2}-1324 a^{2} \gamma+3531 a^{2}-2944 a b \gamma t+2336 a b t+5616 b^{2} t^{2}}{11522 b}} \\
& =\frac{5671\left(\gamma^{2}+1\right)}{4652 \gamma^{2}-1324 \gamma+3531-2944 \gamma\left(t /\left(\frac{a}{b}\right)\right)+2336\left(t /\left(\frac{a}{b}\right)\right)+5616\left(t /\left(\frac{a}{b}\right)\right)^{2}}
\end{aligned}
$$

It has the following expression for $C_{F}=0,1<\gamma \leq 2$, and $\frac{9(\gamma-1) a}{30 b} \leq t \leq \frac{(\gamma-1) a}{2 b}$ :

$$
\begin{aligned}
P_{o} A_{N o T_{P}}^{(B)}(\gamma, t) & =\frac{\frac{\left(\gamma^{2}+1\right) a^{2}}{2 b}}{\frac{11 a^{2} \gamma^{2}-4 a^{2} \gamma+12 a^{2}+4 a b \gamma t+8 a b t-4 b^{2} t^{2}}{32 b}} \\
& =\frac{16\left(\gamma^{2}+1\right)}{11 \gamma^{2}-4 \gamma+12+4 \gamma\left(t /\left(\frac{a}{b}\right)\right)+8\left(t /\left(\frac{a}{b}\right)\right)-4\left(t /\left(\frac{a}{b}\right)\right)^{2}}
\end{aligned}
$$

(vi) For the second modification of the third game with Nash bargaining in the first stage, the price of anarchy under the Bentham global welfare model has the following expression for $C_{F}=0,1<\gamma \leq 2$, and $0 \leq t<\frac{3 \gamma a}{14 b}$ :

$$
\begin{aligned}
\operatorname{PoA}_{N a s h B}^{(B)}(\gamma, t) & =\frac{\frac{\left(\gamma^{2}+1\right) a^{2}}{2 b}}{\frac{\frac{11}{26}(\gamma a)^{2}-\frac{6}{13}(\gamma a)(b t)+\frac{23}{26}(b t)^{2}+\frac{3}{8} a^{2}-\frac{1}{26}(\gamma a) a-\frac{2}{13} a(b t)}{b}} \\
& =\frac{\gamma^{2}+1}{\frac{11}{13} \gamma^{2}-\frac{12 \gamma+4}{13}\left(t /\left(\frac{a}{b}\right)\right)+\frac{23}{13}\left(t /\left(\frac{a}{b}\right)\right)^{2}+\frac{3}{4}-\frac{1}{13} \gamma}
\end{aligned}
$$

Remark 6.4 As already mentioned, $\operatorname{Po} A_{N o P T T(N o D)}^{(B)}$ is a constant. Moreover, by using formula (28), one can also see that $\operatorname{PoA}_{N o P T T(D)}^{(B)}(\gamma)$ is a decreasing function of $\gamma \in(1,+\infty)$,

$$
\lim _{\gamma \rightarrow 1^{+}} \operatorname{Po} A_{N o P T T(D)}^{(B)}(\gamma)=\frac{32}{19}
$$

and

$$
\lim _{\gamma \rightarrow+\infty} \operatorname{Po} A_{N o P T T(D)}^{(B)}(\gamma)=\frac{4}{3}
$$

Similarly, by exploiting formula (29), one can see that

$$
\lim _{\gamma \rightarrow 1^{+}} \operatorname{PoA}_{P T T}^{(B)}(\gamma, t)= \begin{cases}\frac{32}{19} & \text { if } t=0, \\ \frac{32}{19} & \text { if } t>0,\end{cases}
$$

(since, for $\gamma$ sufficiently close to 1 , one gets $t_{l}=0$, and $t_{h} \simeq 0$ ), and that, for each fixed $t \geq 0$,

$$
\lim _{\gamma \rightarrow+\infty} \operatorname{Po} A_{P T T}^{(B)}(\gamma, t)=\frac{9}{8} .
$$


By exploiting formula (30), one can see that, for $0 \leq t<\frac{3 \gamma a}{14 b}$,

$$
\lim _{\gamma \rightarrow 1^{+}} \operatorname{Po} A_{P T O}^{(B)}(\gamma, t)=\frac{1}{\frac{79}{104}-\frac{8}{13}\left(t /\left(\frac{a}{b}\right)\right)+\frac{23}{26}\left(t /\left(\frac{a}{b}\right)\right)^{2}},
$$

and

$$
\operatorname{PoA}_{P T O}^{(B)}(2, t)=\frac{1}{\frac{249}{13}-\frac{64}{65}\left(t /\left(\frac{a}{b}\right)\right)+\frac{23}{65}\left(t /\left(\frac{a}{b}\right)\right)^{2}} .
$$

By exploiting formulas (31) and (32), one can see that, for $0 \leq t<\frac{9(\gamma-1) a}{30 b}$,

$$
\lim _{\gamma \rightarrow 1^{+}} \operatorname{PoA}_{N o T_{P}}^{(B)}(\gamma, t)=\frac{1}{6859-608\left(t /\left(\frac{a}{b}\right)\right)+5616\left(t /\left(\frac{a}{b}\right)\right)^{2}},
$$

and

$$
\operatorname{Po}_{N o T_{P}}^{(B)}(2, t)=\frac{28355}{3\left(6497-1184\left(t /\left(\frac{a}{b}\right)\right)+1872\left(t /\left(\frac{a}{b}\right)\right)^{2}\right)},
$$

and, for $\frac{9(\gamma-1) a}{30 b} \leq t \leq \frac{(\gamma-1) a}{2 b}$,

$$
\lim _{\gamma \rightarrow 1^{+}} \operatorname{PoA}_{N o T_{P}}^{(B)}(\gamma, t)=\frac{104}{79-64\left(t /\left(\frac{a}{b}\right)\right)+92\left(t /\left(\frac{a}{b}\right)\right)^{2}},
$$

and

$$
\operatorname{PoA}_{N o T_{P}}^{(B)}(2, t)=\frac{20}{12+4\left(t /\left(\frac{a}{b}\right)\right)-\left(t /\left(\frac{a}{b}\right)\right)^{2}} .
$$

Finally, by formula (33), the behavior of $\operatorname{Po}_{N a s h B}^{(B)}(\gamma, t)$ is identical to that of $\operatorname{Po} A_{P T O}^{(B)}(\gamma, t)$.

The following result extends Proposition 6.1 to the general case $C_{F} \geq 0$ and shows also a monotonicity property of the price of anarchy with respect to $C_{F}$. In the following, we use the subscript $X$ to refer to one of the six models (i.e., $X=N o P T T(N o D), N o P T T(D), P T T, P T O, N o T_{P}$, and $\left.N a s h B\right)$, whereas, for each such $X, f_{D, X}\left(\gamma, t /\left(\frac{a}{b}\right)\right)$ denotes the function defined as the ratio between the first occurrence of the denominator in the correspondent case among formulas (27)-(33), and $\frac{a^{2}}{b}$. More precisely, one has

$$
\begin{aligned}
& f_{D, N o P T T(N o D)}\left(\gamma, t /\left(\frac{a}{b}\right)\right)=\frac{3\left(\gamma^{2}+1\right)}{8} \\
& f_{D, N o P T T(D)}\left(\gamma, t /\left(\frac{a}{b}\right)\right)=\frac{12 \gamma^{2}+7}{32}, \\
& f_{D, P T T}\left(\gamma, t /\left(\frac{a}{b}\right)\right)= \begin{cases}\frac{32 \gamma^{2}-4 \gamma-1-4\left(t /\left(\frac{a}{b}\right)\right)^{2}-4(2 \gamma+1)\left(t /\left(\frac{a}{b}\right)\right)}{72} & \text { if } 0 \leq t<t_{l}, \\
\frac{124 \gamma^{2}-44 \gamma+91-32\left(t /\left(\frac{a}{b}\right)\right)^{2}+8(5-2 \gamma)\left(t /\left(\frac{a}{b}\right)\right)}{288} & \text { if } t_{l} \leq t \leq t_{h}, \\
\frac{12 \gamma^{2}+7}{32} & \text { if } t>t_{h},\end{cases} \\
& f_{D, P T O}\left(\gamma, t /\left(\frac{a}{b}\right)\right)=\frac{11}{26} \gamma^{2}-\frac{6 \gamma+2}{13}\left(t /\left(\frac{a}{b}\right)\right)+\frac{23}{26}\left(t /\left(\frac{a}{b}\right)\right)^{2}+\frac{3}{8}-\frac{1}{26} \gamma \\
& f_{D, N o T_{P}}\left(\gamma, t /\left(\frac{a}{b}\right)\right)=\frac{4652 \gamma^{2}-1324 \gamma+3531-2944 \gamma\left(t /\left(\frac{a}{b}\right)\right)+2336\left(t /\left(\frac{a}{b}\right)\right)+5616\left(t /\left(\frac{a}{b}\right)\right)^{2}}{11522}
\end{aligned}
$$


for $0 \leq t<\frac{9(\gamma-1) a}{30 b}$

$$
f_{D, N o T_{P}}\left(\gamma, t /\left(\frac{a}{b}\right)\right)=\frac{11}{32} \gamma^{2}-\frac{1}{8} \gamma+\frac{3}{8}+\frac{1}{8} \gamma\left(t /\left(\frac{a}{b}\right)\right)+\frac{1}{4}\left(t /\left(\frac{a}{b}\right)\right)-\frac{1}{8}\left(t /\left(\frac{a}{b}\right)\right)^{2}
$$

for $\frac{9(\gamma-1) a}{30 b} \leq t \leq \frac{(\gamma-1)}{2 b}$, and

$$
f_{D, N a s h B}\left(\gamma, t /\left(\frac{a}{b}\right)\right)=\frac{11}{26} \gamma^{2}-\frac{6 \gamma+2}{13}\left(t /\left(\frac{a}{b}\right)\right)+\frac{23}{26}\left(t /\left(\frac{a}{b}\right)\right)^{2}+\frac{3}{8}-\frac{1}{26} \gamma .
$$

Likewise for Proposition 6.1, an interesting consequence of the next Proposition 6.2 is that the price of anarchy is a function only of $\gamma$ and $C_{F} /\left(\frac{a^{2}}{b}\right)$ for the first two models, whereas, for the other four models, it is a function only of $\gamma, C_{F} /\left(\frac{a^{2}}{b}\right)$, and $t /\left(\frac{a}{b}\right)$.

Proposition 6.2 (i) For each of the six models $X$ in the paper $\left(X=N o P T T(N o D), N o P T T(D), P T T, P T O, N o T_{P}\right.$, and $N a s h B)$ and $C_{F} \geq 0$, the price of anarchy under the Bentham global welfare model can be expressed as

$$
P o A_{X}^{(B)}\left(\gamma, t, C_{F}\right)=\frac{\max \left\{\frac{a^{2}}{b} f_{N}(\gamma)-C_{F}, 0\right\}}{\max \left\{\frac{a^{2}}{b} f_{D, X}\left(\gamma, t /\left(\frac{a}{b}\right)\right)-C_{F}, 0\right\}},
$$

where $f_{N}(\gamma)=\frac{\gamma^{2}+1}{2}$, whereas $0<f_{D, X}\left(\gamma, t /\left(\frac{a}{b}\right)\right) \leq f_{N}(\gamma)$, and $f_{D, X}\left(\gamma, t /\left(\frac{a}{b}\right)\right)$ depends on the model, according to formulas (44)-(50).

(ii) The price of anarchy $\operatorname{Po}_{X}^{(B)}\left(\gamma, t, C_{F}\right)$ is:

1. nondecreasing in $C_{F}$ for $0 \leq C_{F}<\frac{a^{2}}{b} f_{D, X}\left(\gamma, t /\left(\frac{a}{b}\right)\right)$;

2. $+\infty$ for $\frac{a^{2}}{b} f_{D, X}\left(\gamma, t /\left(\frac{a}{b}\right)\right) \leq C_{F}<\frac{a^{2}}{b} f_{N}(\gamma)$;

3. 1 for $C_{F} \geq \frac{a^{2}}{b} f_{N}(\gamma)$.

The three cases in Proposition 6.2 (ii) correspond, respectively, to the situations in which:

- both the manufacturer and the global planner agree on entering the market;

- the manufacturer decides not to enter the market and disagrees with the global planner;

- they both agree on not entering the market

(see also the end of Remark 4.1). In particular, in the last two cases, the price of anarchy is, respectively, equal to $+\infty$ and to 1. Instead, for a small total fixed cost of market entry, the price of anarchy is nondecreasing with respect to it.

Remark 6.5 For each model $X$ in Proposition 6.2, the expression $\frac{a^{2}}{b} f_{D, X}\left(\gamma, t /\left(\frac{a}{b}\right)\right)$ in formula (51) represents the maximum value of $C_{F}$ for which the manufacturer chooses to enter the market. Hence, a comparison of the expressions $\frac{a^{2}}{b} f_{D, X}\left(\gamma, t /\left(\frac{a}{b}\right)\right)$ for the various models allows one to identify, for any choice of the set of parameters, which model provides the largest incentive for the manufacturer to enter the market (see also Remark 5.8). An equivalent comparison can be obtained by comparing the prices of anarchy associated with the various models. Indeed, according to Proposition 6.2, the model with the largest incentive for the manufacturer to enter the market is the one having the largest threshold value $\frac{a^{2}}{b} f_{D, X}\left(\gamma, t /\left(\frac{a}{b}\right)\right)$ on $C_{F}$ for which the associated price of anarchy becomes infinite (in case $\left.\frac{a^{2}}{b} f_{D, X}\left(\gamma, t /\left(\frac{a}{b}\right)\right)<f_{N}(\gamma)\right)$.

Finally, we mention that all the results presented in this section about the price of anarchy are extended straightforwardly to the case in which the total fixed cost $C_{F}$ is a random variable whose realization becomes known to the manufacturer (and also to the global planner) after taking the decision whether to enter or not to enter the market, and the global welfare is replaced by its expected value. In this situation, indeed, in case the manufacturer decides to enter the market, one can simply replace the (deterministic) total fixed cost $C_{F}$ in various formulas (such as (11) and (14)) by the realization of the random total fixed cost of market entry. This case is dealt with numerically in Section 7. 


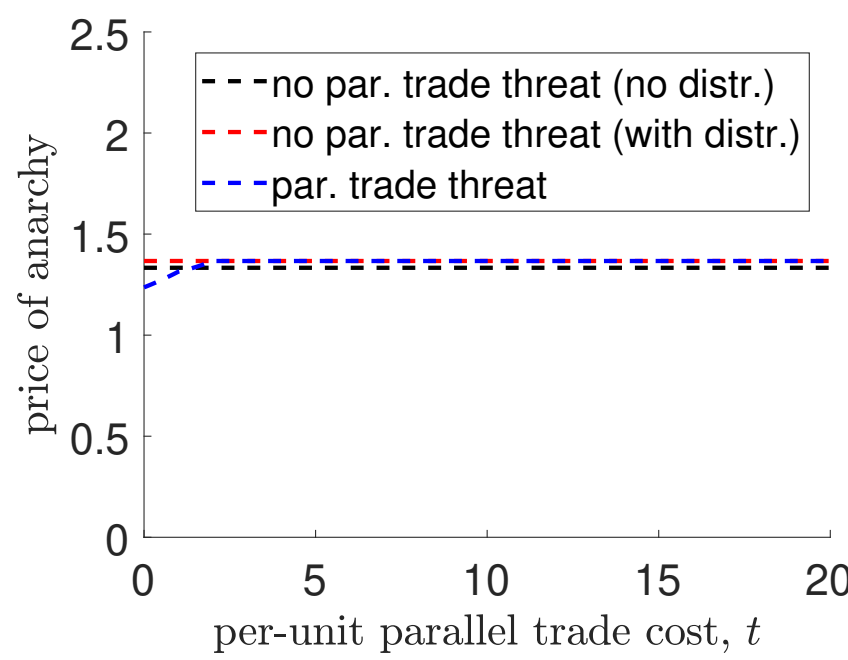

(a)

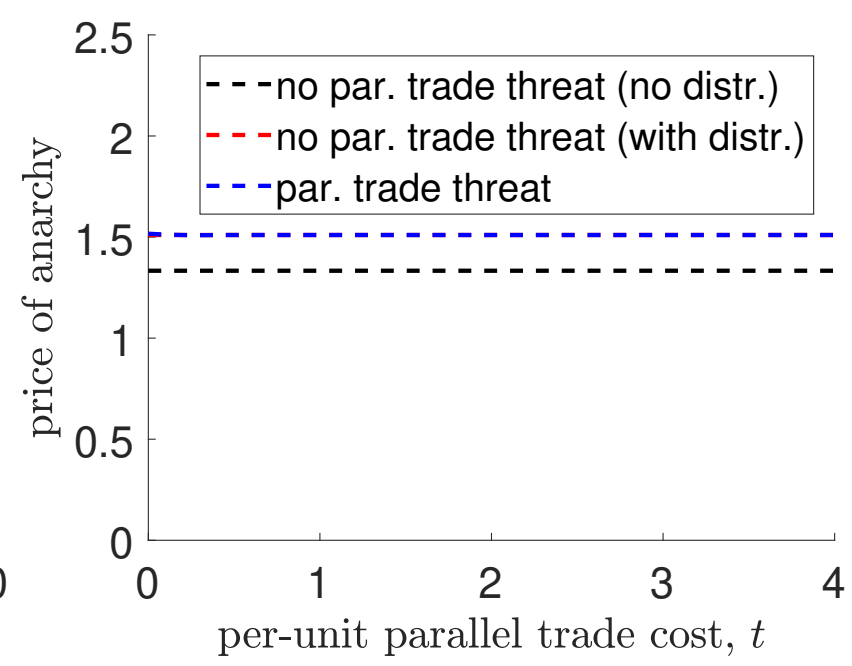

(b)

Fig. 2: Plots of the prices of anarchy (as functions of the parallel trade cost per-unit $t$ ) for the first three models examined in the paper, for: (a) $a=4 / 3, b=1, \gamma=4, C_{F}=0$, and (b) $a=4 / 3, b=1, \gamma=1.6, C_{F}=0$ (for interpretation of the references to color in this figure caption, the reader is referred to the web version of this paper).

\section{Numerical Results}

Figure 2 (a) and (b) shows the behavior of the price of anarchy (as a function of the parallel trade cost per-unit $t$ ) for the first three models examined in the paper, for two choices of the set of parameters $a, b$, and $\gamma$, and for $C_{F}=0$ (the case $C_{F}>0$ is considered later in this section). A MATLAB 7.7.0 implementation has been used to generate the figures in this section. Figure 2 (a) and (b) refers only to the cases of the first three models, since $\gamma=4>2$ has been chosen to generate the plots in the first subfigure, whereas the conditions $0 \leq t<\frac{3 \gamma a}{14 b}, 0 \leq t<\frac{9(\gamma-1) a}{30 b}$, and $\frac{9(\gamma-1) a}{30 b} \leq t \leq \frac{(\gamma-1) a}{2 b}$ hold only for a small range of values for $t$ in the second subfigure (however, numerical results related also to the other three models are provided later in Figure 3). In Figure 2 (a), the price of anarchy for the model with no parallel trade threat and no distributor is typically smaller than the ones for the other two models, apart for very small values of $t$, for which the model of parallel trade threat has a slightly smaller price of anarchy. However, all these prices are close to 1, indicating that the corresponding optimal solutions/subgame-perfect Nash equilibria are quite efficient, and no change in any model (due, e.g., to the possible intervention of a policymaker) is really needed to improve the efficiency significantly. In Figure 2 (b), which refers to a different choice for $\gamma$, the model with no parallel trade threat and no distributor has always the smallest price of anarchy, whereas the other two models have very similar prices of anarchy. Also in this case, the various prices of anarchy are quite close to 1.

We now consider the case $C_{F}>0$. Figure 3 shows, under various assumptions on $C_{F}>0$, the optimal value of the global welfare for the global planner and, for each model considered in the paper, the value of the global welfare at any optimal solution/subgame-perfect Nash equilibrium, and the correspondent price of anarchy. All these quantities are plotted as functions of the parallel trade cost per-unit $t$, and the parameters $a, b, \gamma$ and $t$ are chosen in such a way that all the models hold simultaneously (recall that, compared with the first three models, the last three models have also the constraint $1<\gamma \leq 2$ and model-dependent constraints on $t$ ). The first two columns in Figure 3 refer to two different values (respectively, 0.8 and 1.1) for a deterministic total fixed cost, whereas the last column refers to a random total fixed cost, assuming the value 0.8 with a-priori probability 0.7 , and 1.1 with a-priori probability 0.3 . For these specific cases, an inspection of Figure 3 and a comparison of the results reported in Sections 5 and 6 reveal the following:

(i) for all the cases considered in the figure, the optimal value of the global welfare for the global planner is the same when parallel trade is permitted/forbidden, confirming that the price of anarchy coincides with the normalized price of anarchy (see Remark 6.2); 
(ii) the optimal value of the global welfare for the global planner is larger for the smaller value of $C_{F}$, and in the stochastic case, it is in between the two values obtained in the deterministic case;

(iii) for the two noncooperative games modeling no parallel trade threat/parallel trade threat, the values of the global welfare at their subgame-perfect Nash equilibria are nearly the same, for each of the three models of $C_{F}$. Moreover, when the parallel trade cost per-unit $t$ is sufficiently large, such values are exactly the same for both games (since for large $t$, the parallel trade threat is not credible);

(iv) the decision-theoretic model with no parallel trade threat and no distributor is always more efficient than the game-theoretic model of no parallel trade threat in the presence of the distributor;

(v) the decision-theoretic model with no parallel trade threat and no distributor and the noncooperative game with Nash bargaining in the first stage have typically the largest values of the global welfare, hence the smallest prices of anarchy. The model of parallel trade occurrence with transfer payment has a similar behavior for small $t$, but for $t$ sufficiently large, its price of anarchy is significantly larger than the ones for the two models above. Hence, in that case, an intervention by a policy maker (e.g., a switch to the model with Nash bargaining in the first stage) would be needed to improve the efficiency significantly. The remaining models have larger prices of anarchy;

(vi) in general, among all the models, the equilibria associated with the "pure" models of parallel trade (see Remark 5.7) have the smallest efficiencies.

Figure 4 reports all the six prices of anarchy for three values of $\gamma$ (respectively, $\gamma=1.2, \gamma=1.5$, and $\gamma=1.8$ ) for which all the models apply, by varying $C_{F}$ and $t$, respectively, in the intervals $\left[0, \frac{a^{2}}{2 b}\right]$ and $\left[0, \min \left\{\frac{3 \gamma a}{14 b}, \frac{(\gamma-1) a}{2 b}\right\}\right)$. In this case, the figure does not specify the values of $a$ and $b$ and reports as axes the "normalized" total fixed cost $C_{F} /\left(\frac{a^{2}}{b}\right)$ and the "normalized" parallel trade cost per-unit $t /\left(\frac{a}{b}\right)$. Likewise Figure 3, also this figure shows that, for nonnegligible $t /\left(\frac{a}{b}\right)$, the decision-theoretic model with no parallel trade threat and no distributor and the noncooperative game with Nash bargaining in the first stage have the smallest prices of anarchy, whereas the largest ones are obtained for the "pure" models of parallel trade. The model of parallel trade occurrence with transfer payment has good efficiency (but not the best one), only for negligible $t /\left(\frac{a}{b}\right)$. It is worth also comparing that model with its two modifications. Figure 4 shows that, in the ranges considered for the parameters, the presence of Nash bargaining in the first stage makes the equilibrium of the second modification of the third game be more efficient than the one of the original model. Instead, the absence of the transfer payment makes the equilibrium of the first modification of the third game be less efficient than the one of the original model.

Finally, Figure 5 illustrates, for each model, the maximum value of $C_{F} /\left(\frac{a^{2}}{b}\right)$ for which the manufacturer has the incentive to enter the market (e.g., to invest in $\mathrm{R} \& \mathrm{D}$, if the total fixed cost of entering the market models the total fixed cost of R \& D), which is computed according to Remark 5.8. To generate the figure, the parameters have been chosen likewise for Figure 4. Figure 5 shows that the decision-theoretic model with no parallel trade threat and no distributor provides the largest incentive because, when compared with the other models, it assumes less constraints on the manufacturer's choice. The game-theoretic model of no parallel trade threat in the presence of the distributor provides a smaller incentive, since in that case the manufacturer does not sell directly to the country $B$, but through the distributor. Hence, in that case, the manufacturer has to take into account the distributor's choice to optimize his own behavior. Finally, the two "pure" models of parallel trade provide typically the smallest incentives (which, in these two cases, decrease when decreasing the parallel trade cost per-unit). However, compared with these two models, larger incentives are obtained by considering the two models of parallel trade that include the transfer payment as a "corrective action", since in those cases the manufacturer is able to take over (part of) the distributor's surplus. Of these last two models, the one with Nash bargaining provides a smaller incentive, since in this case the manufacturer can take over only part of such surplus, whereas in the other model the manufacturer can take over the whole distributor's surplus. Interestingly, for this reason, in the latter model the incentive to enter the market is not monotonic with respect to the parallel trade cost per-unit. 


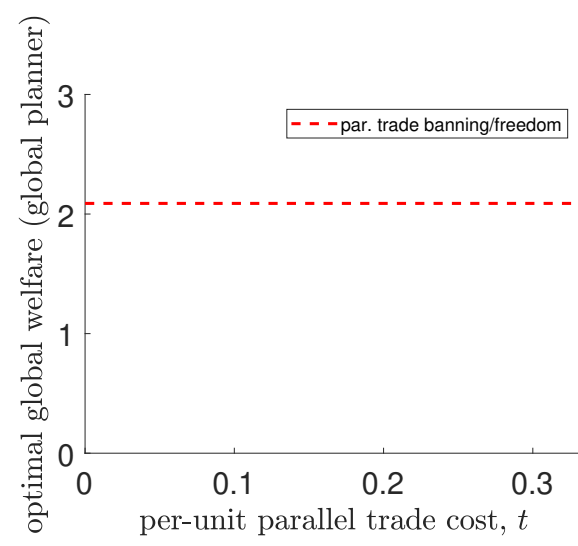

(a)

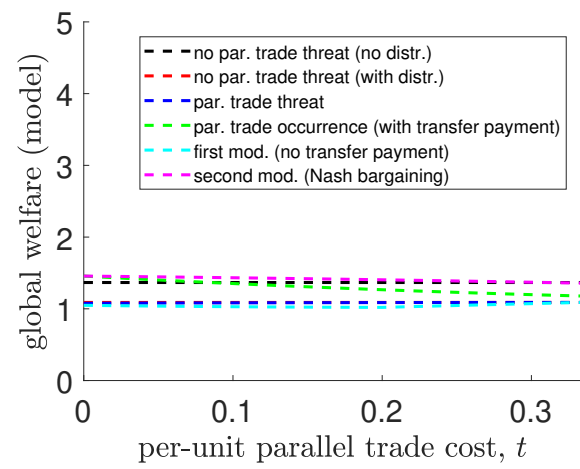

(d)

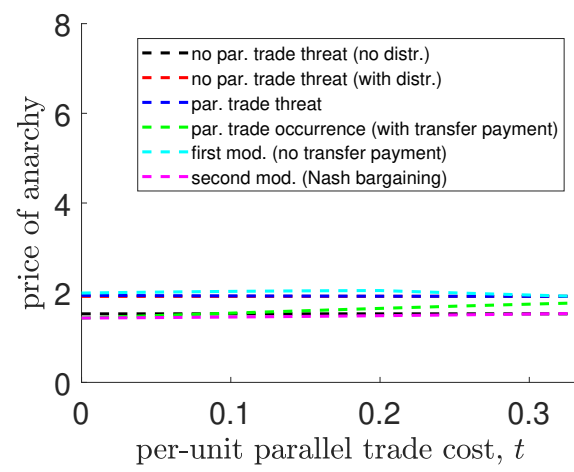

(g)

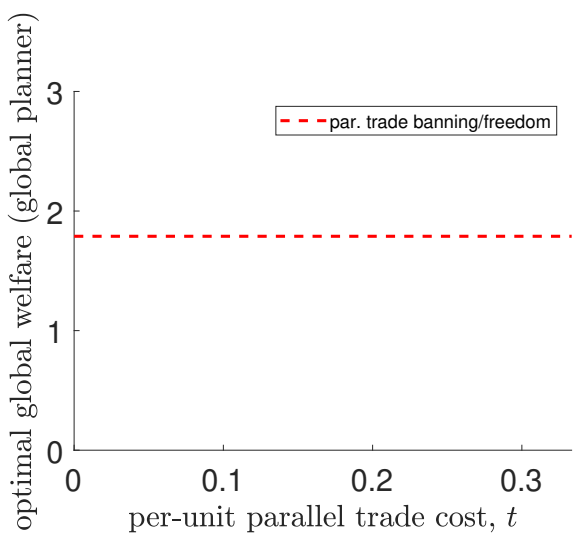

(b)

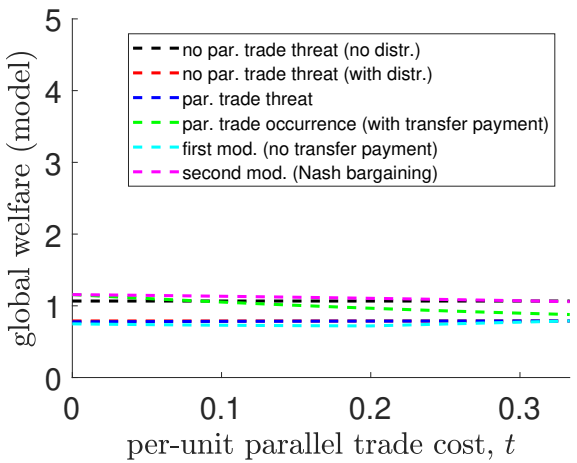

(e)

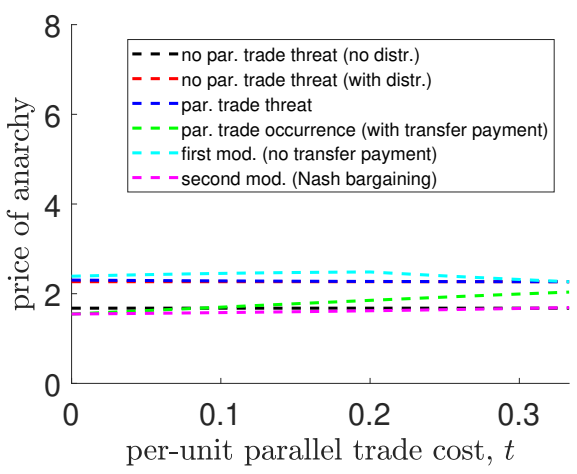

(h)

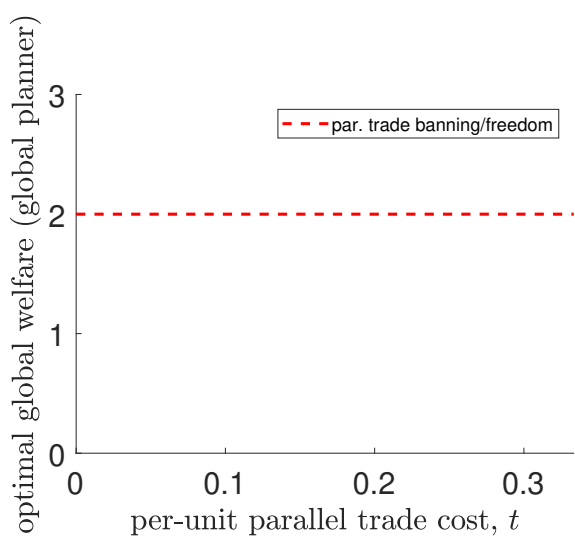

(c)

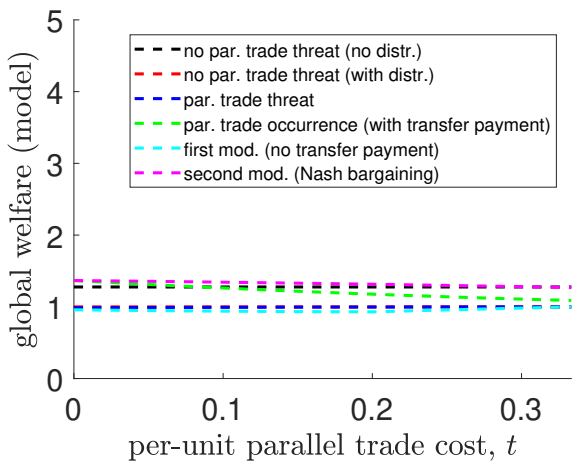

(f)

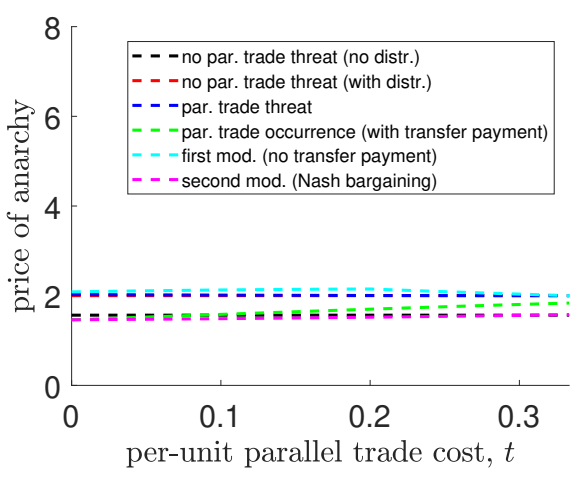

(i)

Fig. 3: For the models examined in the paper: plots (as functions of the parallel trade cost per-unit $t$ ) of (a)-(c) the optimal value of the global welfare for the global planner; (d)-(f) the value of the global welfare at any optimal solution/subgame-perfect Nash equilibrium; (g)-(i) the price of anarchy. The total fixed cost is $C_{F}=0.8$ in the first column, $C_{F}=1.1$ in the second column, whereas in the first third $C_{F}$ is a random variable assuming the value 0.8 with a-priori probability 0.7 , and 1.1 with a-priori probability 0.3 . The values for the other parameters in all the subfigures are $a=4 / 3, b=1$, and $\gamma=1.5$ (for interpretation of the references to color in this figure caption, the reader is referred to the web version of this paper).

\section{Conclusions}

The price of anarchy is a useful tool to measure the efficiency of both equilibrium solutions to noncooperative games and optimal solutions to decision-theoretic models. Although originally proposed in [26] for the case of Nash equilibria, it can be applied also to its refinements (e.g., to subgame-perfect Nash equilibria) and to other solution concepts. In practice, when the price of anarchy for a specific model is "large", this means that the model itself should be changed, in order to obtain a much more efficient solution. Instead, when it is "small", no change of rules by a policymaker (such as a government) is really needed to improve the efficiency significantly. 


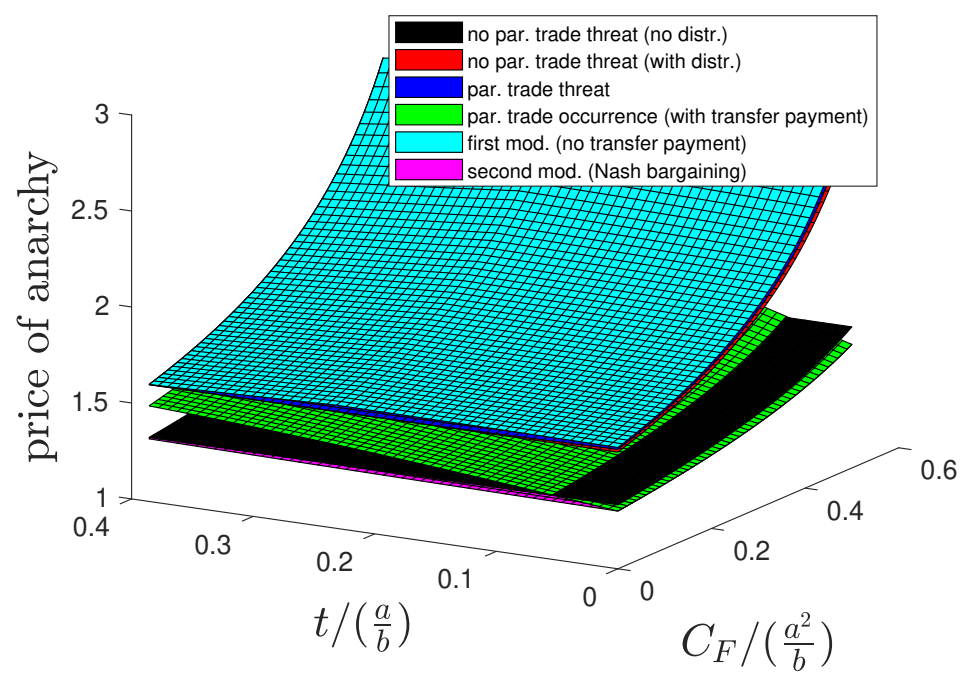

(a) $\gamma=1.2$

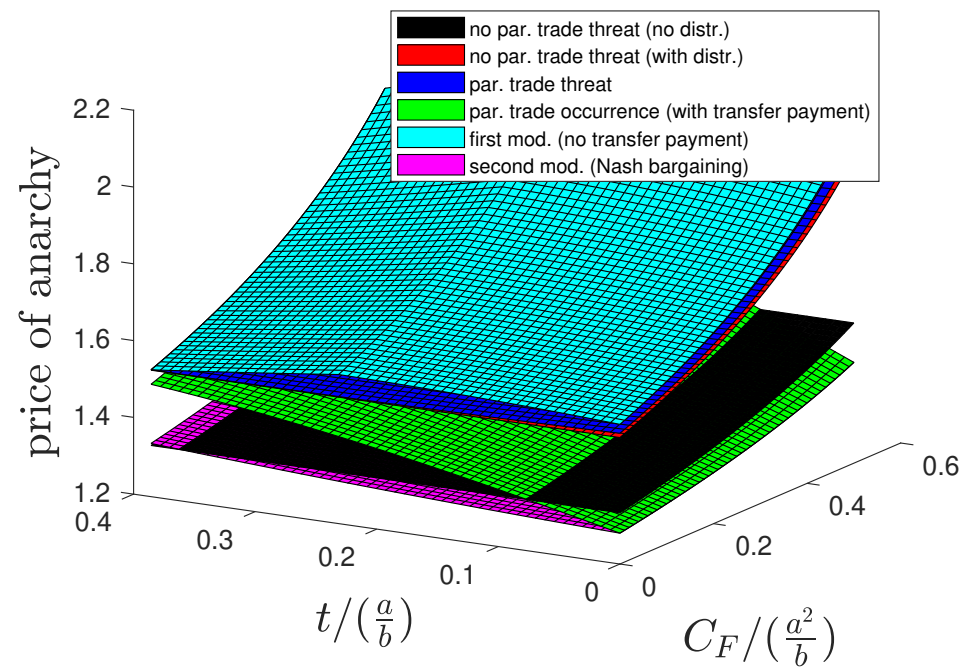

(b) $\gamma=1.5$

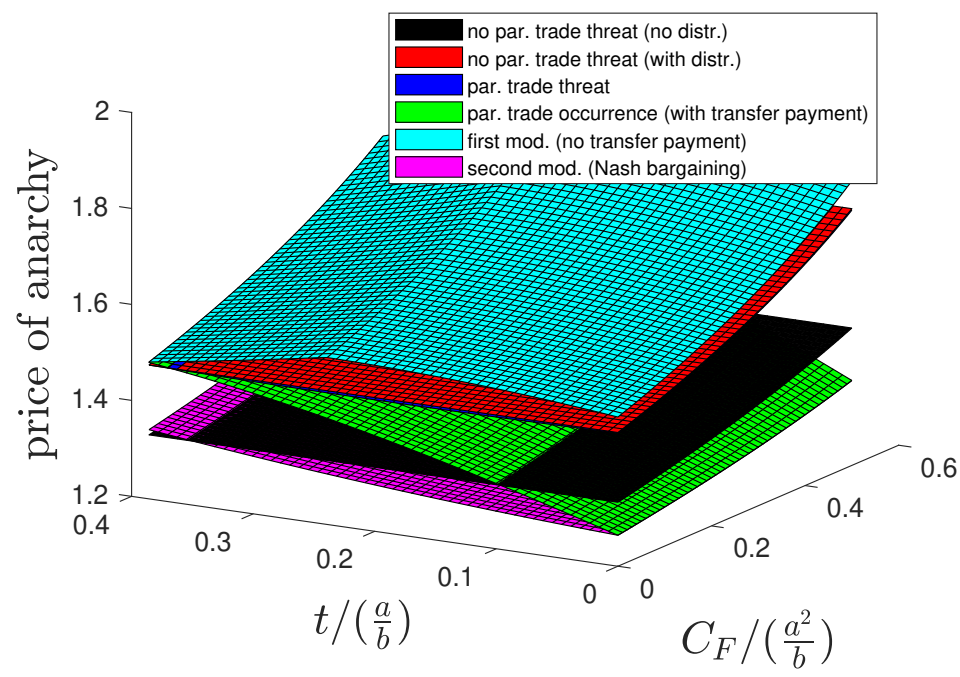

(c) $\gamma=1.8$

Fig. 4: For all the models examined in the paper: plots (as functions of the normalized total fixed $\operatorname{cost} C_{F} /\left(\frac{a^{2}}{b}\right)$ and the normalized parallel trade cost per-unit $\left.t /\left(\frac{a}{b}\right)\right)$ of the price of anarchy. The three cases (a), (b), and (c) refer, respectively, to the choices $\gamma=1.2, \gamma=1.5$, and $\gamma=1.8$ for the relative market size (for interpretation of the references to color in this figure caption, the reader is referred to the web version of this paper). 


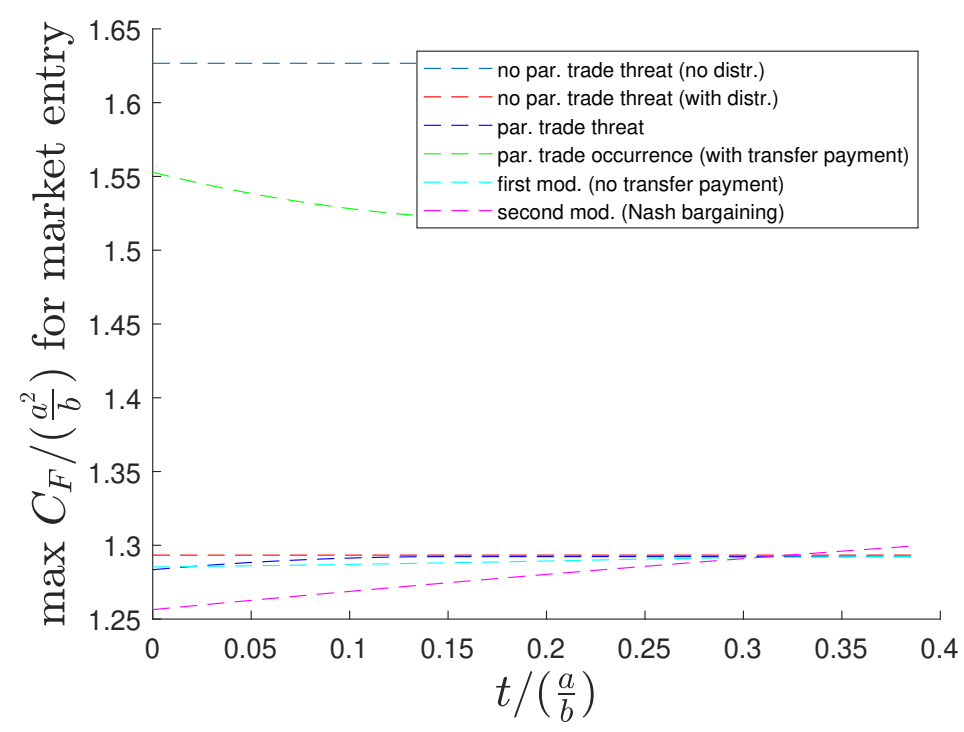

(a) $\gamma=1.2$

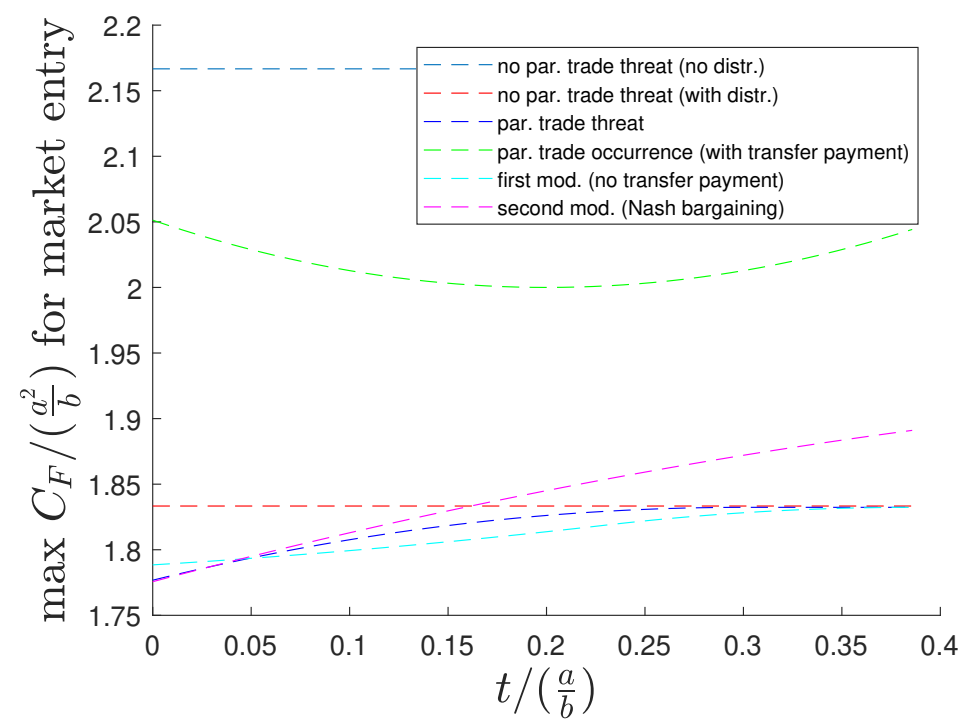

(b) $\gamma=1.5$

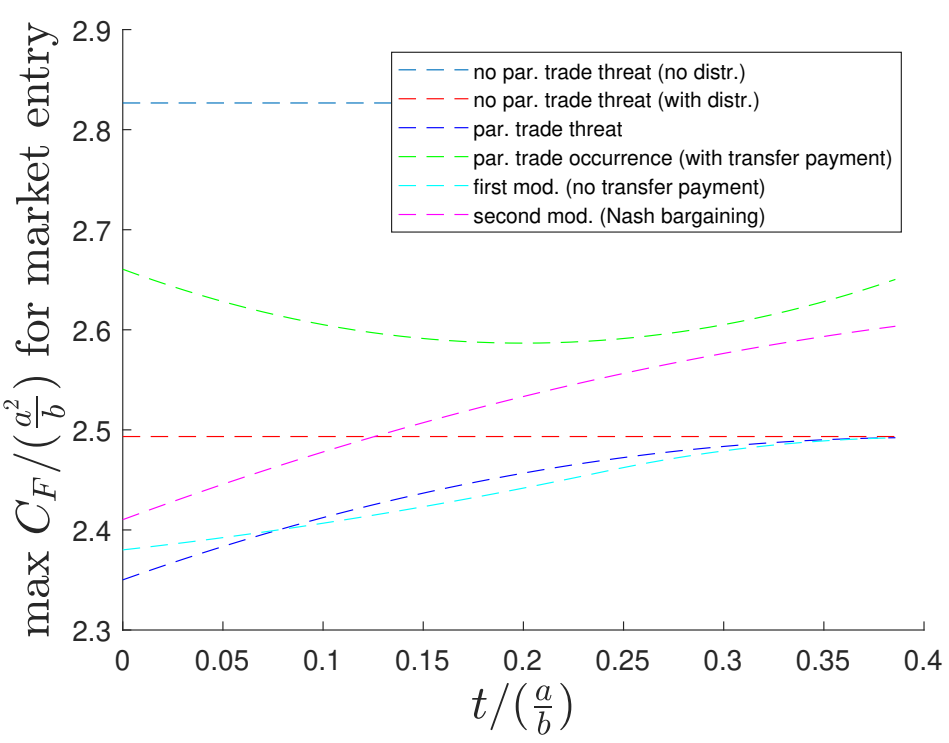

(c) $\gamma=1.8$

Fig. 5: For all the models examined in the paper: plots (as functions of the normalized parallel trade cost per-unit $\left.t /\left(\frac{a}{b}\right)\right)$ of the maximum value of $C_{F} /\left(\frac{a^{2}}{b}\right)$ for which the manufacturer has the incentive to enter the market. The three cases (a), (b), and (c) refer, respectively, to the choices $\gamma=1.2, \gamma=1.5$, and $\gamma=1.8$ for the relative market size (for interpretation of the references to color in this figure caption, the reader is referred to the web version of this paper). 
In the paper, we have applied the price of anarchy to the investigation and comparison of some models (i.e., a decision-theoretic model, and five noncooperative game-theoretic models) associated with different levels of parallel trade freedom. We have also compared such models with respect to the manufacturer's incentive to invest in Research and Development ( $\mathrm{R} \& \mathrm{D})$, and more generally, the incentive to enter the market. Such comparisons have been motivated by the negative effects of parallel trade on both efficiency and the manufacturer's incentive to invest in $\mathrm{R}$ \& D, which were already highlighted in several theoretical and empirical works, such as [9-11, 14, 23, 28, 32, 45]. In the models examined in this paper, the price of anarchy measures the ratio between the optimal value of the global welfare and its value obtained in correspondence of the "worst" solution for each specific model. In the analysis, the marginal cost of production has been neglected. This assumption is reasonable for sectors characterized by a large total fixed cost of $\mathrm{R} \& \mathrm{D}$, such as the pharmaceutical, software and music recording industries [31].

First, in the paper we have formulated and solved in closed form suitable quadratic optimization problems modeling the decision-making process of a global planner, whose aim is to maximize the global welfare under suitable constraints. Second, we have obtained closed-form expressions for the price of anarchy for several models of the interaction between a manufacturer and a distributor possibly involved in parallel trade of pharmaceuticals. The models have been ordered according to an increasing level of complexity of the interaction between the manufacturer and the distributor. The obtained expressions of the price of anarchy are sensitive to the values of the relative market size of the two countries and (up to suitable normalizations) of the parallel trade cost per-unit and of the total fixed cost of R \& D. The prices of anarchy for the various models have been also evaluated numerically, including the case of a random total fixed cost of R \& D. The results of our numerical comparison show that, in the ranges considered for the parameters, the models of parallel trade threat/occurrence that do not include the transfer payment have the largest prices of anarchy (i.e., their equilibria have the smallest efficiencies), and also provide typically the smallest incentives for the manufacturer to invest in R \& D. However, from a policy-making perspective, improvements are obtained if suitable countermeasures are taken to help the manufacturer recover from the costs of $\mathrm{R} \& \mathrm{D}$, such as the inclusion of a transfer payment in the model. The results are in agreement with several studies (see, e.g., $[10,11,45]$ ), which highlight the negative effects of parallel trade, and the need to take suitable countermeasures.

An interesting feature of the results of our analysis is that it was possible to find in closed form the optimal solutions of both the quadratic optimization problems used to study the global planner's optimal behavior (i.e., when maximizing the global welfare $)^{15}$, and also the prices of anarchy associated with the various models. The models investigated in the paper could be applied to specific empirical case studies after identifying the values of their parameters $a, b, \gamma$, $t$, and $C_{F}$ (and in the stochastic case, also the a-priori probability distribution of $C_{F}$ ). In principle, the evaluation of the price of anarchy may be extended to other choices of the global welfare function and to nonlinear demand functions, possibly including in the models also a non-zero constant marginal cost of production. However, in these cases, differently from the situations considered in this paper, it may be difficult to obtain closed-form expressions. Moreover, an extension of the analysis could be obtained by inserting a feedback from the total fixed cost of $\mathrm{R} \& \mathrm{D}$ into the demand functions, i.e., making the parameters $a, b$, and $\gamma$ be functions of $C_{F}$, and modeling $C_{F}$ itself as a decisional variable ${ }^{16}$. In this case, one should take into account that the global planner and the manufacturer could have different optimal total fixed costs of $\mathrm{R} \& \mathrm{D}$. Hence, for this modification, one would obtain expressions for the prices of anarchy similar to the ones contained in Proposition 6.2, but with $C_{F}$ in formula (51) replaced by two possibly different endogeneously determined total fixed costs of $\mathrm{R} \& \mathrm{D}$, respectively at its numerator and at its denominator. Moreover, in this case, the prices of anarchy would not be necessarily nondecreasing with respect to the total fixed cost of R \& D. As another possible extension, the price of anarchy could be evaluated also for other models of parallel trade, such as the ones studied in $[8,20,22,33]$, which are other noncooperative games for which parallel trade actually

\footnotetext{
15 Since closed-form optimal solutions were obtained, it was not necessary to use in the analysis more advanced tools from quadratic programming [5].

16 For instance, one could assume that $\gamma$ increases as $C_{F}$ increases. This would correspond to the perception of a larger quality of the product (e.g., as a consequence of a larger effort in R \& D) by the consumers in the country where the manufacturer is located.
} 
occurs at equilibrium when it is permitted ${ }^{17}$, and for their possible extensions including the multi-country case, supply quota restrictions, currency differences, and the presence of competitor drugs. The results of such a comparison would be useful to measure the efficiency of the proposed solutions, and to detect when policymakers should change the rules of each game in order to increase the value of the global welfare significantly. Similarly, one could compare the manufacturers' incentive to invest in $\mathrm{R} \& \mathrm{D}$ for the various cases.

Appendix: Proofs

Proof of formula (5). In order to prove the formula, let us suppose that, of the total $q_{A}=q_{A}^{M, C}+q_{A}^{D, C}$ units of the product sold to the consumers in the country $A$, the first $q_{A}^{M, C}$ ones are sold by the manufacturer, whereas the last $q_{A}^{D, C}$ ones are sold by the distributor. Then, taking into account the expression (1) of the demand function in the country $A$, the consumers' surplus in the country $A$ for the first $q_{A}^{M, C}$ units of the product is

$$
S_{C_{A}}^{(I)}=\int_{0}^{q_{A}^{M, C}}\left(\frac{\gamma a-q}{b}-p_{A}^{M, C}\right) d q=\left(\frac{\gamma a}{b}-p_{A}^{M, C}\right) q_{A}^{M, C}-\frac{\left(q_{A}^{M, C}\right)^{2}}{2 b} .
$$

Similarly, the consumers' surplus in the country $A$ for the remaining $q_{A}^{D, C}$ units of the product is

$$
\begin{aligned}
S_{C_{A}}^{(I I)} & =\int_{q_{A}^{M, C}}^{q_{A}^{M, C}+q_{A}^{D, C}}\left(\frac{\gamma a-q}{b}-p_{A}^{D, C}\right) d q \\
& =\left(\frac{\gamma a}{b}-p_{A}^{D, C}\right) q_{A}^{D, C}-\frac{\left(q_{A}^{M, C}+q_{A}^{D, C}\right)^{2}}{2 b}+\frac{\left(q_{A}^{M, C}\right)^{2}}{2 b}
\end{aligned}
$$

Then, formula (5) is obtained by summing (52) and (53).

It is important to observe that one obtains exactly the same expression (5) for $S_{C_{A}}$ if other choices for the seller are considered, for each unit of the product. For instance, if one assumes instead that the first $q_{A}^{D, C}$ units of the product bought by the consumers in the country $A$ are sold by the distributor, whereas the last $q_{A}^{M, C}$ ones are sold by the manufacturer, formulas (52) and (53) are replaced, respectively, by

$$
S_{C_{A}}^{\left(I^{\prime}\right)}=\int_{0}^{q_{A}^{D, C}}\left(\frac{\gamma a-q}{b}-p_{A}^{D, C}\right) d q=\left(\frac{\gamma a}{b}-p_{A}^{D, C}\right) q_{A}^{D, C}-\frac{\left(q_{A}^{D, C}\right)^{2}}{2 b},
$$

and

$$
\begin{aligned}
S_{C_{A}}^{\left(I I^{\prime}\right)} & =\int_{q_{A}^{D, C}}^{q_{A}^{D, C}+q_{A}^{M, C}}\left(\frac{\gamma a-q}{b}-p_{A}^{M, C}\right) d q \\
& =\left(\frac{\gamma a}{b}-p_{A}^{M, C}\right) q_{A}^{M, C}-\frac{\left(q_{A}^{D, C}+q_{A}^{M, C}\right)^{2}}{2 b}+\frac{\left(q_{A}^{D, C}\right)^{2}}{2 b},
\end{aligned}
$$

and also the sum of (54) and (55) provides the same expression (5) for $S_{C_{A}}$.

Proof of Proposition 4.1. We start considering the case in which the manufacturer enters the market. One can observe that the global welfare $G W_{P T F}^{(B)}$ provided by formula (8) is a function having the separable structure

$$
G W_{P T F}^{(B)}\left(C_{F}, q_{A}^{M, C}, q_{A}^{D, C}, q_{B}^{D, C}\right)=G W_{P T F}^{(B, I)}\left(q_{A}^{M, C}, q_{A}^{D, C}\right)+G W_{P T F}^{(B, I I)}\left(q_{B}^{D, C}\right)
$$

\footnotetext{
17 For complex models, function approximators may be combined with backward induction to find approximations of their equilibria, likewise in $[15,19]$, where different problems having a single decision maker were considered.
} 
where

$$
G W_{P T F}^{(B, I)}\left(C_{F}, q_{A}^{M, C}, q_{A}^{D, C}\right)=\frac{\gamma a}{b}\left(q_{A}^{M, C}+q_{A}^{D, C}\right)-\frac{\left(q_{A}^{M, C}+q_{A}^{D, C}\right)^{2}}{2 b}-t q_{A}^{D, C}-C_{F},
$$

and

$$
G W_{P T F}^{(B, I I)}\left(q_{B}^{D, C}\right)=\frac{a}{b} q_{B}^{D, C}-\frac{\left(q_{B}^{D, C}\right)^{2}}{2 b} .
$$

Hence, due to the separability of its objective function and to the form of its constraints, solving the optimization problem (10) is reduced to solving the two following optimization problems:

$$
\begin{aligned}
& \underset{q_{A}^{M, C}, q_{A}^{D, C}}{\operatorname{maximize}} G W_{P T F}^{(B, I)}\left(C_{F}, q_{A}^{M, C}, q_{A}^{D, C}\right)=\frac{\gamma a}{b}\left(q_{A}^{M, C}+q_{A}^{D, C}\right)-\frac{\left(q_{A}^{M, C}+q_{A}^{D, C}\right)^{2}}{2 b}-t q_{A}^{D, C}-C_{F} \\
& \text { s.t. } \quad q_{A}^{M, C}, q_{A}^{D, C} \geq 0,
\end{aligned}
$$

and

$$
\begin{aligned}
& \underset{q_{B}^{D, C}}{\operatorname{maximize}} G W_{P T F}^{(B, I I)}\left(q_{B}^{D, C}\right)=\frac{a}{b} q_{B}^{D, C}-\frac{\left(q_{B}^{D, C}\right)^{2}}{2 b} \\
& \text { s.t. } \quad q_{B}^{D, C} \geq 0 .
\end{aligned}
$$

We now consider the two problems separately.

(i) The optimization problem (59) is a concave quadratic maximization problem. By introducing the Lagrangian function

$$
L^{(I)}\left(C_{F}, q_{A}^{M, C}, q_{A}^{D, C}, \mu_{A}^{M, C}, \mu_{A}^{D, C}\right)=G W_{P T F}^{(B, I)}\left(C_{F}, q_{A}^{M, C}, q_{A}^{D, C}\right)+\mu_{A}^{M, C} q_{A}^{M, C}+\mu_{A}^{D, C} q_{A}^{D, C},
$$

it is solved by imposing the following Karush-Kuhn-Tucker optimality conditions ${ }^{18}$.

$$
\left\{\begin{array}{lrl}
\text { stationarity : } & \frac{\partial L^{(I)}}{\partial q_{A}^{M, C}}=\frac{\gamma a}{b}-\frac{q_{A}^{M, C}+q_{A}^{D, C}}{b}+\mu_{A}^{M, C} & =0, \\
& \frac{\partial L^{(I)}}{\partial q_{A}^{D, C}}=\frac{\gamma a}{b}-\frac{q_{A}^{M, C}+q_{A}^{D, C}}{b}-t+\mu_{A}^{D, C} & =0, \\
\text { primal feasibility : } & q_{A}^{M, C}, q_{A}^{D, C} \geq 0, \\
\text { dual feasibility : } & \mu_{A}^{M, C}, \mu_{A}^{D, C} \geq 0, \\
\text { complementary slackness : } & \mu_{A}^{M, C} q_{A}^{M, C}, \mu_{A}^{D, C} q_{A}^{D, C}=0 .
\end{array}\right.
$$

Then, it is straightforward to see that, for any $t>0$, the system (62) has the unique solution

$$
\left\{\begin{array}{l}
q_{A}^{M, C}=\gamma a, \\
q_{A}^{D, C}=0, \\
\mu_{A}^{M, C}=0, \\
\mu_{A}^{D, C}=t .
\end{array}\right.
$$

\footnotetext{
18 For both optimization problems (59) and (60), the qualification of the constraints holds, due to their linearity [3]. Moreover, for both problems, Karush-Kuhn-Tucker optimality conditions [29] are necessary and sufficient for optimality, due to the concavity of the respective objective functions.
} 
For $t=0$, one gets the infinite number of solutions described by

$$
\left\{\begin{array}{l}
q_{A}^{M, C}, q_{A}^{D, C} \geq 0 \text { s.t. } q_{A}^{M, C}+q_{A}^{D, C}=\gamma a, \\
\mu_{A}^{M, C}=0, \\
\mu_{A}^{D, C}=0 .
\end{array}\right.
$$

Finally, for both formulas (63) and (64), one has $q_{A}=q_{A}^{M, C}+q_{A}^{D, C}=\gamma a$. Hence, for both cases, the value of the objective $G W_{P T F}^{(B, I)}\left(C_{F}, q_{A}^{M, C}, q_{A}^{D, C}\right)$ at optimality is

$$
\left(G W_{P T F}^{(B, I)}\right)^{\circ}=\frac{(\gamma a)^{2}}{b}-C_{F}-\frac{(\gamma a)^{2}}{2 b}=\frac{(\gamma a)^{2}}{2 b}-C_{F} .
$$

(ii) Similarly, also the optimization problem (60) is a concave quadratic maximization problem. Again, by introducing the Lagrangian function

$$
L^{(I I)}\left(q_{B}^{D, C}, \mu_{B}^{D, C}\right)=\frac{a}{b} q_{B}^{D, C}-\frac{\left(q_{B}^{D, C}\right)^{2}}{2 b},
$$

also the optimization problem (60) is solved by imposing the Karush-Kuhn-Tucker optimality conditions, which have now the following form:

$$
\left\{\begin{array}{lrl}
\text { stationarity }: & \frac{\partial L^{(I I)}}{\partial q_{B}^{D, C}}=\frac{a}{b}-\frac{q_{B}^{D, C}}{b}+\mu_{B}^{D, C} & =0 \\
\text { primal feasibility : } & q_{B}^{D, C} \geq 0 \\
\text { dual feasibility : } & \mu_{B}^{D, C} \geq 0 \\
\text { complementary slackness : } & \mu_{B}^{D, C} q_{B}^{D, C}=0
\end{array}\right.
$$

Then, one can see that, for any $t \geq 0$, the system (67) has the unique solution

$$
\left\{\begin{array}{l}
q_{B}^{D, C}=a \\
\mu_{B}^{D, C}=0
\end{array}\right.
$$

Finally, the value of the objective $G W_{P T F}^{(B, I)}\left(q_{B}^{D, C}\right)$ at optimality is

$$
\left(G W_{P T F}^{(B, I I)}\right)^{\circ}=\frac{a^{2}}{b}-\frac{a^{2}}{2 b}=\frac{a^{2}}{2 b} .
$$

Concluding, the optimal value of the objective $G W_{P T F}^{(B)}\left(C_{F}, q_{A}^{M, D}, q_{A}^{D, C}, q_{B}^{D, C}\right)$ of the original optimization problem (10) modeling parallel trade freedom under the Bentham global welfare model is

$$
\left(G W_{P T F}^{(B)}\right)^{\circ}=\left(G W_{P T F}^{(B, I)}\right)^{\circ}+\left(G W_{P T F}^{(B, I I)}\right)^{\circ}=\frac{\left(\gamma^{2}+1\right) a^{2}}{2 b}-C_{F},
$$

which is (11).

Finally, for the case in which the manufacturer does not enter the market, (12) follows directly from the definition of the Bentham global welfare function.

Proof of Proposition 4.2. As shown in the proof of Proposition 4.1, for any $t \geq 0$, among the optimal solutions of the optimization problem (10), there is always one for which $q_{A}^{D, C}=0$, which is feasible for the more constrained optimization problem (13), in the sense that it satisfies all its constraints. Hence, one obtains (14), whereas (15) follows directly from the definition of the Bentham global welfare function. 
Proof of Proposition 5.1. The result follows from formulas (3), (5), and (6), and from the following expressions for the prices and quantities at the optimal solution of the manufacturer's surplus maximization problem for the specific model, which were proved in [34, Section 4.1]:

$$
\begin{cases}\left(p_{A}^{M, C}\right)^{\circ, N o P T T(N o D)} & =\frac{\gamma a}{2 b} \\ \left(q_{A}^{M, C}\right)^{\circ, N o P T T(N o D)} & =\frac{\gamma a}{2} \\ \left(p_{B}^{M, C}\right)^{\circ, N o P T T(N o D)} & =\frac{a}{2 b} \\ \left(q_{B}^{M, C}\right)^{\circ, N o P T T(N o D)} & =\frac{a}{2} .\end{cases}
$$

Proof of Proposition 5.2. The result follows from formulas (3), (4), (5), and (6), and from the following expressions for the prices and quantities at any subgame-perfect Nash equilibrium of the game modeling no parallel trade threat in the presence of the distributor, which were proved in [36]:

$$
\left\{\begin{aligned}
\left(p_{A}^{M, C}\right)^{(\text {s.p.Nash }, N o P T T(D))} & =\frac{\gamma a}{2 b} \\
\left(p_{A}^{D, C}\right)^{(\text {s.p.Nash }, N o P T T(D))} & =(\text { not uniquely determined, but irrelevant as } \\
& \left.\left(q_{A}^{D, C}\right)^{(\text {s.p.Nash }, N o P T T(D))}=0\right) \\
\left(p_{B}^{M, D}\right)^{(\text {s.p.Nash }, N o P T T(D))} & =\frac{a}{2 b} \\
\left(p_{B}^{D, C}\right)^{(\text {s.p.Nash }, N o P T T(D))} & =\frac{3 a}{4 b} \\
\left(q_{A}^{M, C}\right)^{(\text {s.p.Nash }, N o P T T(D))} & =\frac{\gamma a}{2} \\
\left(q_{A}^{D, C}\right)^{(\text {s.p.Nash }, N o P T T(D))} & =0 \\
\left(q_{B}^{M, D}\right)^{(\text {s.p.Nash,NoPTT(D)) }} & =\frac{a}{4} \\
\left(q_{B}^{D, C}\right)^{(\text {s.p.Nash }, N o P T T(D))} & =\frac{a}{4} .
\end{aligned}\right.
$$

Proof of Proposition 5.3. Also in this case, the result follows from formulas (3), (4), (5), and (6), and from the following expressions for the prices and quantities at any subgame-perfect Nash equilibrium of the game modeling parallel trade threat, which were proved in [36]: 
if $0 \leq t<t_{l}:\left\{\begin{aligned}\left(p_{A}^{M, C}\right)^{(\text {s.p.Nash }, l, P T T)}= & \frac{a}{6 b}(2 \gamma+1)+\frac{t}{3}, \\ \left(p_{A}^{D, C}\right)^{(\text {s.p.Nash }, l, P T T)}= & (\text { not uniquely determined, but irrelevant as } \\ & \left.\left(q_{A}^{D, C}\right)^{(\text {s.p.Nash }, l, P T T)}=0\right), \\ \left(p_{B}^{M, D}\right)^{(\text {s.p.Nash }, l, P T T)}= & (\text { not uniquely determined, but irrelevant as } \\ & \left.\left(q_{B}^{M, D}\right)^{(\text {s.p.Nash }, l, P T T)}=0\right), \\ \left(p_{B}^{D, C}\right)^{(\text {s.p.Nash }, l, P T T)}= & (\text { not uniquely determined, but irrelevant as } \\ & \left.\left(q_{B}^{D, C}\right)^{(\text {s.p.Nash }, l, P T T)}=0\right), \\ \left(q_{A}^{M, C}\right)^{(\text {s.p.Nash }, l, P T T)}= & \frac{a}{6}(4 \gamma-1)-\frac{b t}{3}, \\ \left(q_{A}^{D, C}\right)^{(\text {s.p.Nash }, l, P T T)}= & 0, \\ \left(q_{B}^{M, D}\right)^{(\text {s.p.Nash }, l, P T T)}= & 0, \\ \left(q_{B}^{D, C}\right)^{(\text {s.p.Nash }, l, P T T)}= & 0,\end{aligned}\right.$

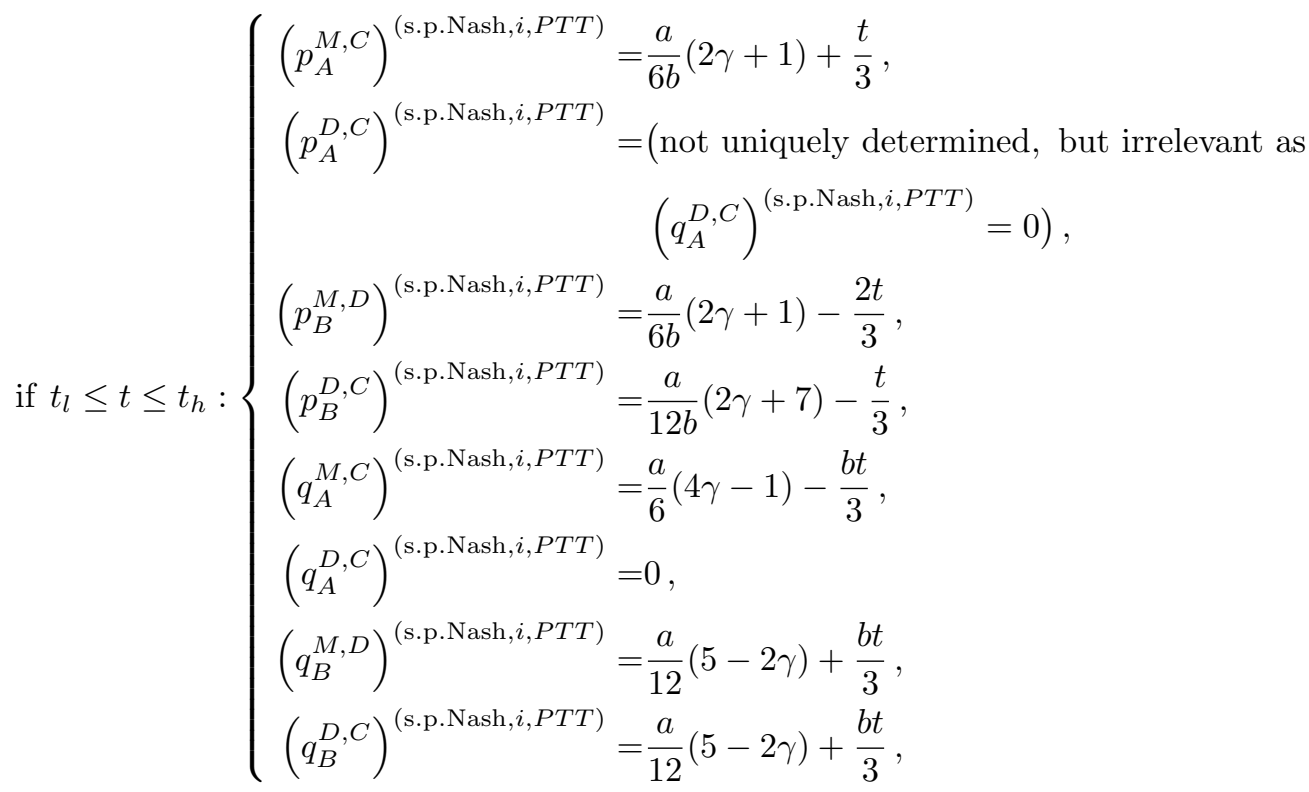




$$
\text { if } t>t_{h}:\left\{\begin{array}{l}
\left(p_{A}^{M, C}\right)^{(\text {s.p.Nash }, h, P T T)}=\frac{\gamma a}{2 b} \\
\left(p_{A}^{D, C}\right)^{(\text {s.p.Nash }, h, P T T)}=(\text { not uniquely determined, but irrelevant as } \\
\left(p_{A}^{M, D}\right)^{(\text {s.p.Nash }, h, P T T)}=\frac{a}{2 b} \\
\left(p_{B}^{D, C}\right)^{(\text {s.p.Nash }, h, P T T)}=\frac{3 a}{4 b} \\
\left(q_{A}^{M, C}\right)^{(\text {s.p.Nash }, h, P T T)}=\frac{\gamma a}{2} \\
\left(q_{A}^{D, C}\right)^{(\text {s.p.Nash }, h, P T T)}=0 \\
\left(q_{B}^{M, D}\right)^{(\text {s.p.Nash }, h, P T T)}=\frac{a}{4} \\
\left(q_{B}^{D, C}\right)^{(\text {s.p.Nash }, h, P T T)}=\frac{a}{4} .
\end{array}\right.
$$

Proof of Proposition 5.4. We recall from [31] that the following expressions hold for the prices and quantities at the unique subgame-perfect Nash equilibrium of the game modeling parallel trade occurrence with transfer payment, under the assumptions $1<\gamma \leq 2$ and $0 \leq t<\frac{3 \gamma a}{14 b}$ (this second condition is needed to ensure the occurrence of parallel trade, i.e., to have $\left(q_{A}^{D, C}\right)^{\text {(s.p.Nash, } P T O)}>0$ in formula $\left.(75)\right)^{19}$ :

$$
\left\{\begin{array}{l}
\left(p_{A}^{M, C}\right)^{(\text {s.p.Nash }, P T O)}=\frac{5 \gamma a+7 b t}{13 b}, \\
\left(p_{A}^{D, C}\right)^{(\text {s.p.Nash }, P T O)}=\frac{5 \gamma a+7 b t}{13 b}, \\
\left(p_{B}^{M, D}\right)^{(\text {s.p.Nash }, P T O)}=\frac{2 \gamma a+8 b t}{13 b}, \\
\left(p_{B}^{D, C}\right)^{(\text {s.p.Nash }, P T O)}=\frac{a}{2 b}+\frac{\gamma a+4 b t}{13 b}, \\
\left(q_{A}^{M, C}\right)^{(\text {s.p.Nash }, P T O)}=\frac{5 \gamma a+7 b t}{13}, \\
\left(q_{A}^{D, C}\right)^{(\text {s.p.Nash }, P T O)}=\frac{3 \gamma a-14 b t}{13}, \\
\left(q_{B}^{M, D}\right)^{(\text {s.p.Nash }, P T O)}=\frac{a}{2}+\frac{2 \gamma a-18 b t}{13} \\
\left(q_{B}^{D, C}\right)^{(\text {s.p.Nash }, P T O)}=\frac{a}{2}-\frac{\gamma a+4 b t}{13} .
\end{array}\right.
$$

The associated transfer payment is

$$
\left(T_{P}\right)^{(\text {s.p.Nash, } P T O)}=\frac{(9 \gamma a-42 b t)^{2}}{1521 b}+\frac{((13-2 \gamma) a-8 b t)^{2}}{676 b}
$$

Finally, the statement is obtained combining the results above with formulas (3), (4), (5), and (6).

Proof of Proposition 5.5. (i) Let $1<\gamma \leq 2$ and $0 \leq t<\frac{9(\gamma-1) a}{30 b}$. Proceeding by backward induction likewise in the proof of [31, Proposition 1] (see, in particular, pages 555 and 556 therein), in the first stage the manufacturer has to

\footnotetext{
19 As already mentioned, the model presented in [31] refers to the choices $a=1$ and $b=1$. For uniformity of notation with respect to the other two models, we have re-done its computations removing the two assumptions $a=1$ and $b=1$, obtaining the results shown in formula (75).
} 
solve the two following optimization problems to determine an equilibrium value for the price $p_{B}^{M, D}$ :

$$
\begin{aligned}
\underset{p_{B}^{M, D}}{\operatorname{maximize}} & \frac{\left(\gamma a+p_{B}^{M, D} b+b t\right)^{2}}{9 b}+p_{B}^{M, D} \frac{\gamma a-2 p_{B}^{M, D} b-2 b t}{3}+p_{B}^{M, D} \frac{a-p_{B}^{M, D} b}{2}-C_{F} \\
\text { s.t. } & 0 \leq p_{B}^{M, D}<\frac{\gamma a-2 b t}{2 b},
\end{aligned}
$$

and

$$
\begin{array}{cl}
\underset{p_{B}^{M, D}}{\operatorname{maximize}} & \frac{\gamma^{2} a^{2}}{4 b}+p_{B}^{M, D} \frac{a-p_{B}^{M, D} b}{2}-C_{F} \\
\text { s.t. } & \frac{\gamma a-2 b t}{2 b} \leq p_{B}^{M, D} \leq \frac{a}{b}
\end{array}
$$

The two optimization problems above correspond to the manufacturer's profit maximization for the cases, respectively, of parallel trade occurrence at equilibrium, and no parallel trade occurrence at equilibrium. An equilibrium value for the price $p_{B}^{M, D}$ provides the largest among the optimal objective values of the two optimization problems. Solving the two optimization problems above, one can verify immediately that, due to the assumption ${ }^{20} 0 \leq t<\frac{9(\gamma-1) a}{30 b}$,

$$
\left(p_{B}^{M, D}\right)^{\left(\mathrm{s} \cdot \mathrm{p} \cdot \mathrm{Nash}, N o T_{P}\right)}=\frac{(10 \gamma+9) a-8 b t}{38 b}
$$

is the unique equilibrium value for $p_{B}^{M, D}$. The other equilibrium prices and quantities are determined via the equilibrium analysis in the second stage (obtained proceeding likewise in [31]). In summary, one obtains the following expressions for the equilibrium values:

$$
\left\{\begin{array}{l}
\left(p_{A}^{M, C}\right)^{\left(\text {s.p.Nash }, N o T_{P}\right)}=\frac{\gamma a+\frac{(10 \gamma+9) a-8 b t}{38 b} b+b t}{3 b} \\
\left(p_{A}^{D, C}\right)^{\left(\text {s.p.Nash }, N o T_{P}\right)}=\frac{\gamma a+\frac{(10 \gamma+9) a-8 b t}{38 b} b+b t}{3 b} \\
\left(p_{B}^{M, D}\right)^{\left(\text {s.p.Nash }, N o T_{P}\right)}=\frac{(10 \gamma+9) a-8 b t}{38 b}, \\
\left(p_{B}^{D, C}\right)^{\left(\text {s.p.Nash }, N o T_{P}\right)}=\frac{a+\frac{(10 \gamma+9) a-8 b t}{38 b} b}{2 b}, \\
\left(q_{A}^{M, C}\right)^{\left(\text {s.p.Nash }, N o T_{P}\right)}=\frac{\gamma a+\frac{(10 \gamma+9) a-8 b t}{38 b} b+b t}{3}, \\
\left(q_{A}^{D, C}\right)^{\left(\text {s.p.Nash }, N o T_{P}\right)}=\frac{\gamma a-2 \frac{(10 \gamma+9) a-8 b t}{38 b} b-2 b t}{3}, \\
\left(q_{B}^{M, D}\right)^{\left(\text {s.p.Nash }, N o T_{P}\right)}=\frac{\gamma a-2 \frac{(10 \gamma+9) a-8 b t}{38 b} b-2 b t}{3}+\frac{a-\frac{(10 \gamma+9) a-8 b t}{38 b} b}{2} \\
\left(q_{B}^{D, C}\right)^{\left(\text {s.p.Nash }, N o T_{P}\right)}=\frac{a-\frac{(10 \gamma+9) a-8 b t}{38 b} b}{2} .
\end{array}\right.
$$

Finally, the statement is obtained combining the results above with formulas (3), (4), (5), and (6).

(ii) Let $1<\gamma \leq 2$ and $\frac{9(\gamma-1) a}{30 b} \leq t \leq \frac{(\gamma-1) a}{2 b}$. Proceeding likewise in the part (i) of the proof and solving the two optimization problems $(77)$ and (78), one can verify immediately that, due to the assumption ${ }^{21} \frac{9(\gamma-1) a}{30 b} \leq t \leq \frac{(\gamma-1) a}{2 b}$,

$$
\left(p_{B}^{M, D}\right)^{\left(\mathrm{s} \cdot \mathrm{p} \cdot \mathrm{Nash}, N o T_{P}\right)}=\frac{\gamma a-2 b t}{2 b}
$$

\footnotetext{
20 In summary, this assumption has been imposed in order to make the first-order unconstrained optimality condition satisfied inside the feasible set for the first optimization problem, and outside the feasible set for the second optimization problem.

21 In summary, this assumption has been imposed in order to make, for each of the two optimization problems, the first-order unconstrained optimality condition satisfied outside the feasible set, thus restricting the optimality analysis to the boundary of the feasible set.
} 
is the unique equilibrium value for $p_{B}^{M, D}$. The other equilibrium prices and quantities are determined via the equilibrium analysis in the second stage (obtained proceeding likewise in [31]). In summary, one obtains the following expressions for the equilibrium values:

$$
\begin{cases}\left(p_{A}^{M, C}\right)^{\left(\text {s.p.Nash }, N o T_{P}\right)} & =\frac{\gamma a}{2 b} \\ \left(p_{A}^{D, C}\right)^{\left(\text {s.p.Nash }, N o T_{P}\right)} & =(\text { not uniquely determined, but irrelevant as } \\ & \left.\left(q_{A}^{D, C}\right)^{\left(\text {s.p.Nash }, N o T_{P}\right)}=0\right) \\ \left(p_{B}^{M, D}\right)^{\left(\text {s.p.Nash }, N o T_{P}\right)} & =\frac{\gamma a-2 b t}{2 b} \\ \left(p_{B}^{D, C}\right)^{\left(\text {s.p.Nash }, N o T_{P}\right)} & =\frac{(2+\gamma) a-2 b t}{4 b} \\ \left(q_{A}^{M, C}\right)^{\left(\text {s.p.Nash }, N o T_{P}\right)} & =\frac{\gamma a}{2}, \\ \left(q_{A}^{D, C}\right)^{\left(\text {s.p.Nash }, N o T_{P}\right)} & =0, \\ \left(q_{B}^{M, D}\right)^{\left(\text {s.p.Nash }, N o T_{P}\right)} & =\frac{(2-\gamma) a+2 b t}{4} \\ \left(q_{B}^{D, C}\right)^{\left(\text {s.p.Nash }, N o T_{P}\right)} & =\frac{(2-\gamma) a+2 b t}{4}\end{cases}
$$

Finally, the statement is obtained combining the results above with formulas (3), (4), (5), and (6).

Proof of Proposition 5.6. Let $1<\gamma \leq 2$ and $0 \leq t<\frac{3 \gamma a}{14 b}$. Proceeding by backward induction likewise in the proof of [31, Proposition 1] (see, in particular, pages 555 and 556 therein), one obtains, in the first stage, the following expressions for the surpluses of the manufacturer and of the distributor (here, their dependence on $p_{B}^{M, D}$ and $T_{P}$ is highlighted, having replaced the equilibrium values of the other prices and quantities - determined by the equilibrium analysis in the second stage - inside such expressions):

(i) for $0 \leq p_{B}^{M, D}<\frac{\gamma a-2 b t}{2 b}, T_{P} \geq 0$, and $\frac{\left(a-p_{B}^{M, D} b\right)^{2}}{4 b}-T_{P}+\frac{\left(\gamma a-2 p_{B}^{M, D} b-2 b t\right)^{2}}{9 b} \geq 0$ :

$$
\begin{gathered}
S_{M}\left(p_{B}^{M, D}, T_{P}\right)=\frac{\left(\gamma a+p_{B}^{M, D} b+b t\right)^{2}}{9 b}+p_{B}^{M, D} \frac{\gamma a-2 p_{B}^{M, D} b-2 b t}{3}+T_{P}+p_{B}^{M, D} \frac{a-p_{B}^{M, D} b}{2}-C_{F}, \\
S_{D}\left(p_{B}^{M, D}, T_{P}\right)=\frac{\left(a-p_{B}^{M, D} b\right)^{2}}{4 b}-T_{P}+\frac{\left(\gamma a-2 p_{B}^{M, D} b-2 b t\right)^{2}}{9 b}
\end{gathered}
$$

(ii) for $\frac{\gamma a-2 b t}{2 b} \leq p_{B}^{M, D} \leq \frac{a}{b}, T_{P} \geq 0$, and $\frac{\left(a-p_{B}^{M, D} b\right)^{2}}{4 b}-T_{P} \geq 0$ :

$$
\begin{gathered}
S_{M}\left(p_{B}^{M, D}, T_{P}\right)=\frac{\gamma^{2} a^{2}}{4 b}+T_{P}+p_{B}^{M, D} \frac{a-p_{B}^{M, D} b}{2}-C_{F} \\
S_{D}\left(p_{B}^{M, D}, T_{P}\right)=\frac{\left(a-p_{B}^{M, D} b\right)^{2}}{4 b}-T_{P}
\end{gathered}
$$

(iii) otherwise:

$$
\begin{gathered}
S_{M}\left(p_{B}^{M, D}, T_{P}\right)=\frac{\gamma^{2} a^{2}}{4 b}-C_{F}, \\
S_{D}\left(p_{B}^{M, D}, T_{P}\right)=0 .
\end{gathered}
$$

By varying $p_{B}^{M, D}$ and $T_{P}$, one obtains the choice set of feasible surpluses for the manufacturer and for the distributor in the first stage of the game. As an example, its shape is reported in Figure 6 (a) for $C_{F}=0, a=b=1, \gamma=1.6374$, 


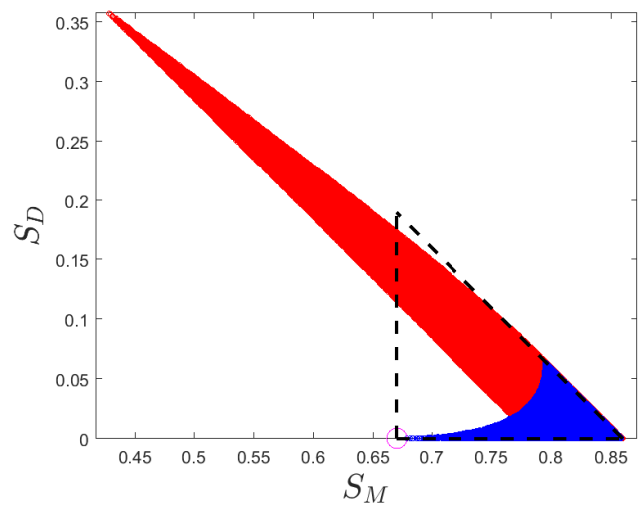

(a)

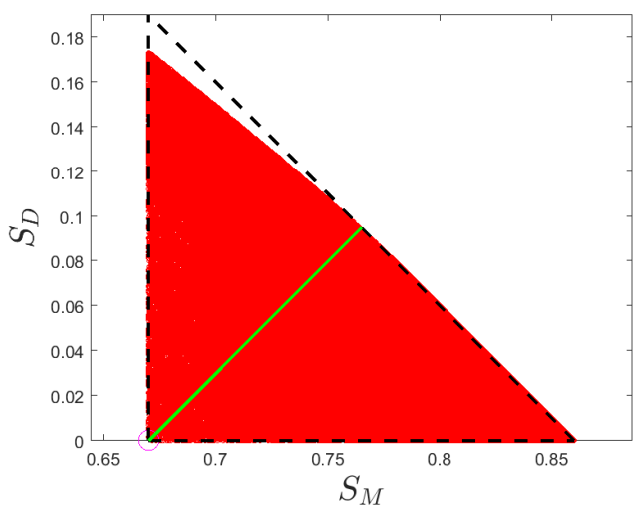

(b)

Fig. 6: (a) Choice set of feasible surpluses for the manufacturer and for the distributor in the first stage of the second modification of the third game-theoretic model, for $C_{F}=0, a=b=1, \gamma=1.6374$, and $t=0.3258$. The regions corresponding to the expressions (i), (ii) and (iii) for the surpluses in the proof of Proposition 5.6 are represented in red, blue, and magenta, respectively. The point represented by the circle in magenta (the third region) is the disagreement point $\left(\frac{\gamma^{2} a^{2}}{4 b}, 0\right)$. The choice set is the intersection of the union of these three regions and the dashed triangle in the figure, since all the points whose value of the horizontal coordinate is smaller than the one of the disagreement point $\left(\frac{\gamma^{2} a^{2}}{4 b}, 0\right)$ can be discarded (because the manufacturer can always choose a retail price $p_{M}^{M, D}$ so high to stay in the third region). (b) The convexification of the choice set (in red). The intersection between its upper boundary and the green line (with slope 1) defines the Nash bargaining solution to the Nash bargaining problem modeling the first stage of the game (for interpretation of the references to color in this figure caption, the reader is referred to the web version of this paper).

and $t=0.3258$. As shown by a preliminary numerical study obtained by varying the parameters $\gamma$ and $t$, this set has indeed the typical shape ${ }^{22}$ reported in Figure 6 (a), and is typically nonconvex ${ }^{23}$. Likewise in [13, Section 6.2], this set can be convexified by randomization, and effectively replaced by its convex hull as the choice set, as reported in Figure 6 (b). As explained in the caption of the figure, the disagreement point is $\left(d_{M}, d_{D}\right):=\left(\frac{\gamma^{2} a^{2}}{4 b}, 0\right)$. Then, the Nash bargaining solution (which maximizes the product $\left(S_{M}-d_{M}\right)\left(S_{D}-d_{D}\right)$ on the convexified choice set; see, e.g., [37, Section 2.4]) is immediately found as the intersection between its upper boundary and the line with slope 1 passing through the disagreement point (see Figure $6(\mathrm{~b})$ ). The reason is that this is the Nash barganining solution for a choice set coincident with the dashed right-angled isosceles triangle in Figure 6 (b), and such a Nash bargaining solution belongs also to the original convexified choice set ${ }^{24}$. In more details, the rightmost point of the convexified choice set is

$$
\left(\frac{\left(\gamma a+\frac{2 \gamma a+8 b t}{13}+b t\right)^{2}}{9 b}+\frac{2 \gamma a+8 b t}{13 b} \frac{\gamma a-2 \frac{2 \gamma a+8 b t}{13}-2 b t}{3}+\frac{\left(\gamma a-2 \frac{2 \gamma a+8 b t}{13}-2 b t\right)^{2}}{9 b}+\frac{a^{2}-\left(\frac{2 \gamma a+8 b t}{13}\right)^{2}}{4 b}-C_{F}, 0\right)
$$

and, as shown in [31], corresponds to the maximum value for the sum of the feasible surpluses of the manufacturer and of the distributor in the first stage, which is obtained for $p_{B}^{M, D}=\frac{2 \gamma a+8 b t}{13 b}$ and $T_{P}=\frac{\left(a-\frac{2 \gamma a+8 b t}{13}\right)^{2}}{4 b}+\frac{\left(\gamma a-2 \frac{2 \gamma a+8 b t}{13}-2 b t\right)^{2}}{9 b}$. The Nash bargaining solution is obtained by decreasing such value of $T_{P}$ by one half the length of the cathethus of

\footnotetext{
22 The reason why, in Figure 6 (a), the upper boundary of the red region is above the upper boundary of the blue region is that in most parts of the figure the former corresponds to the maximum value for the sum of the feasible surpluses of the manufacturer and of the distributor in the first stage, as shown in [31] for its rightmost point under the assumptions of Proposition 5.6. Moreover, its slope is equal to -1 in most parts of the figure because, starting from the value of $T_{P}$ associated with the rightmost point, utility can be transferred from the manufacturer to the distributor, by decreasing $T_{P}$ (until it becomes equal to 0).

23 This is often the case for choice sets of feasible surpluses arising from certain duopoly models [4,42,44].

24 To reach these two conclusions, we have applied the following properties satisfied by the Nash bargaining solution (see, e.g., [37, Section 2.6]): the axioms of Pareto-efficiency and symmetry to find the Nash bargaining solution on the triangle, then the axiom of independence of irrelevant alternatives to find it on the original convexified choice set.
} 
the triangle in Figure 6 (b), i.e., by

$$
\begin{gathered}
\Delta T_{P} \\
=\frac{1}{2}\left[\frac{\left(\gamma a+\frac{2 \gamma a+8 b t}{13}+b t\right)^{2}}{9 b}+\frac{2 \gamma a+8 b t}{13 b} \frac{\gamma a-2 \frac{2 \gamma a+8 b t}{13}-2 b t}{3}+\frac{\left(\gamma a-2 \frac{2 \gamma a+8 b t}{13}-2 b t\right)^{2}}{9 b}+\frac{a^{2}-\left(\frac{2 \gamma a+8 b t}{13}\right)^{2}}{4 b}-\frac{\gamma^{2} a^{2}}{4 b}\right],
\end{gathered}
$$

and also provides the maximum value for the sum of the feasible surpluses of the manufacturer and of the distributor.

The other equilibrium prices and quantities are determined via the equilibrium analysis in the second stage (obtained proceeding likewise in [31]). In summary, one obtains the following expressions for the equilibrium values:

$$
\left\{\begin{aligned}
\left(p_{A}^{M, C}\right)^{(\text {s.p.Nash }, N a s h B)} & =\frac{\gamma a+\frac{2 \gamma a+8 b t}{13 b} b+b t}{3 b}, \\
\left(p_{A}^{D, C}\right)^{(\text {s.p.Nash }, N a s h B)} & =\frac{\gamma a+\frac{2 \gamma a+8 b t}{13 b} b+b t}{3 b}, \\
\left(p_{B}^{M, D}\right)^{(\text {s.p.Nash }, N a s h B)} & =\frac{2 \gamma a+8 b t}{13 b}, \\
\left(p_{B}^{D, C}\right)^{(\text {s.p.Nash }, N a s h B)} & =\frac{a+\frac{2 \gamma a+8 b t}{13 b} b}{2 b}, \\
\left(q_{A}^{M, C}\right)^{(\text {s.p.Nash }, N a s h B)} & =\frac{\gamma a+\frac{2 \gamma a+8 b t}{13 b} b+b t}{3}, \\
\left(q_{A}^{D, C}\right)^{(\text {s.p.Nash }, N a s h B)} & =\frac{\gamma a-2 \frac{2 \gamma a+8 b t}{13 b} b-2 b t}{3}, \\
\left(q_{B}^{M, D}\right)^{(\text {s.p.Nash }, N a s h B)} & =\frac{\gamma a-2 \frac{2 \gamma a+8 b t}{13 b} b-2 b t}{3}+\frac{a-\frac{(2 \gamma+8 t) a}{13 b} b}{2}, \\
\left(q_{B}^{D, C}\right)^{(\text {s.p.Nash }, N a s h B)} & =\frac{a-\frac{2 \gamma a+8 b t}{13 b} b}{2} . \\
\left(T_{P}\right)^{(\text {s.p.Nash }, N a s h B)} & =\frac{\left(a-\frac{2 \gamma a+8 b t}{13}\right)^{2}}{4 b}+\frac{\left(\gamma a-2 \frac{2 \gamma a+8 b t}{13}-2 b t\right)^{2}}{9 b}-\Delta T_{P}
\end{aligned}\right.
$$

Finally, the statement is obtained combining the results above with formulas (3), (4), (5), and (6).

Proof of Proposition 6.1. (i) For the model without the distributor, the value of the global welfare associated with the optimal solution provided by formula (70) has the following expression for $C_{F}=0$ (such an expression is derived by using formulas (9) and (16)):

$$
G W_{P T B}^{(B, N o P T T(N o D))}(\gamma)=\frac{3 a^{2}}{8 b}\left(\gamma^{2}+1\right)
$$

Then, (27) is derived by applying (92), Proposition 4.2, and Definition 6.1.

(ii) For the game modeling no parallel trade threat in the presence of the distributor, the value of the global welfare associated with the subgame-perfect Nash equilibrium provided by formula (71) has the following expression for $C_{F}=0$ (such an expression is derived by using formulas (9) and (17)):

$$
G W_{P T B}^{(B, \text { s.p.Nash, NoPTT(D)) }}(\gamma)=\frac{a^{2}}{32 b}\left(12 \gamma^{2}+7\right) .
$$

Then, (28) is derived by applying (93), Proposition 4.2 , and Definition 6.1 .

(iii) For the game modeling parallel trade threat, the values of the global welfare associated with the subgame-perfect Nash equilibria provided by formulas (72), (73), and (74) have the following expressions for $C_{F}=0$ (such expressions 
are derived by using formulas (8), (20), (21), and (22)):

$$
\begin{aligned}
G W_{P T F}^{(B, \text { s.p.Nash }, l, P T T)}(\gamma, t) & =\frac{a^{2}}{72 b}\left(32 \gamma^{2}-4 \gamma-1\right)-\frac{1}{18}\left(b t^{2}+a t+2 \gamma a t\right), \\
G W_{P T F}^{(B, \text { s.p.Nash }, i, P T T)}(\gamma, t) & =\frac{a^{2}}{288 b}\left(124 \gamma^{2}-44 \gamma+91\right)-\frac{1}{36}\left(4 b t^{2}+2 \gamma a t-5 a t\right), \\
G W_{P T F}^{(B, \text { s.p.Nash }, h, P T T)}(\gamma, t) & =\frac{a^{2}}{32 b}\left(12 \gamma^{2}+7\right) .
\end{aligned}
$$

Then, (29) is derived by applying (94), Proposition 4.1, and Definition 6.1.

(iv) For the game modeling parallel trade occurrence with transfer payment, the value of the global welfare associated with the subgame-perfect Nash equilibrium provided by formulas (75) and (76) has the following expression for $C_{F}=0$, $1<\gamma \leq 2$, and $0 \leq t<\frac{3 \gamma a}{14 b}$ (such an expression is derived by using formulas (8) and (23)):

$$
\begin{aligned}
G W_{P T F}^{(B, \text { s.p.Nash,PTO })}(\gamma, t)= & \frac{\left(\gamma a+\frac{2 \gamma a+8 b t}{13}+b t\right)^{2}}{9 b}+\frac{2 \gamma a+8 b t}{13 b} \frac{\gamma a-2 \frac{2 \gamma a+8 b t}{13}-2 b t}{3} \\
& +\frac{\left(\gamma a-2 \frac{2 \gamma a+8 b t}{13}-2 b t\right)^{2}}{9 b}+\frac{a^{2}-\left(\frac{2 \gamma a+8 b t}{13}\right)^{2}}{4 b} \\
& +\frac{\left(\gamma a-\frac{5 \gamma a+7 b t}{13}\right)^{2}}{2 b}+\frac{\left(\frac{a}{2}-\frac{\gamma a+4 b t}{13}\right)^{2}}{2 b} \\
= & \frac{\frac{11}{26}(\gamma a)^{2}-\frac{6}{13}(\gamma a)(b t)+\frac{23}{26}(b t)^{2}+\frac{3}{8} a^{2}-\frac{1}{26}(\gamma a) a-\frac{2}{13} a(b t)}{b} .
\end{aligned}
$$

Then, (30) is derived by applying (95), Proposition 4.1, and Definition 6.1.

(v) For the first modification of the third noncooperative game-theoretic model, the value of the global welfare associated with the subgame-perfect Nash equilibrium provided by formulas (80) and (82) has the following expression for $C_{F}=0,1<\gamma \leq 2$, and $0 \leq t<\frac{9(\gamma-1) a}{30 b}$ (such an expression is derived by using formulas (8) and (24)):

$$
G W_{P T F}^{\left(B, \text { s.p.Nash }, N o T_{P}\right)}(\gamma, t)=\frac{4652 a^{2} \gamma^{2}-1324 a^{2} \gamma+3531 a^{2}-2944 a b \gamma t+2336 a b t+5616 b^{2} t^{2}}{11522 b} .
$$

It has the following expression for $C_{F}=0,1<\gamma \leq 2$, and $\frac{9(\gamma-1) a}{30 b} \leq t \leq \frac{(\gamma-1) a}{2 b}$ (such an expression is derived by using formulas (8) and (25)):

$$
\left.G W_{P T F}^{(B, \text { s.p.Nash, NoT }} T_{P}\right)(\gamma, t)=\frac{11 a^{2} \gamma^{2}-4 a^{2} \gamma+12 a^{2}+4 a b \gamma t+8 a b t-4 b^{2} t^{2}}{32 b} .
$$

Then, (31) and (32) are derived by applying (96), (97), Proposition 4.1, and Definition 6.1.

(vi) For the second modification of the third noncooperative game-theoretic model, one obtains exactly the same values for the global welfare and for the price of anarchy as in the case (iv), due to formula (26).

Proof of Proposition 6.2. (i) This follows from the definition of the price of anarchy and its expressions reported in formulas (27)-(33) of Proposition 6.1 for $C_{F}=0$. For the general case $C_{F} \geq 0$, these have the form (51), since one has to take into account the decision (either by the global planner or by the manufacturer) whether to enter or not to enter the market.

(ii) This part of the statement is obtained by examining all the combinations of cases for which the maximum in each of the two expressions $\max \left\{\frac{a^{2}}{b} f_{N}(\gamma)-C_{F}, 0\right\}$ and $\max \left\{\frac{a^{2}}{b} f_{D, X}\left(\gamma, t /\left(\frac{a}{b}\right)\right)-C_{F}, 0\right\}$, is obtained in correspondence 
either of the first or of the second term. In particular, the first case in (ii) is obtained by observing that, for $0 \leq C_{F}^{(1)}<$ $C_{F}^{(2)}$, the inequality

$$
\frac{\frac{a^{2}}{b} f_{N}(\gamma)-C_{F}^{(1)}}{\frac{a^{2}}{b} f_{D, X}\left(\gamma, t /\left(\frac{a}{b}\right)\right)-C_{F}^{(1)}} \leq \frac{\frac{a^{2}}{b} f_{N}(\gamma)-C_{F}^{(2)}}{\frac{a^{2}}{b} f_{D, X}\left(\gamma, t /\left(\frac{a}{b}\right)\right)-C_{F}^{(2)}},
$$

is equivalent to

$$
\left(C_{F}^{(1)}-C_{F}^{(2)}\right)\left(f_{N}(\gamma)-f_{D, X}\left(\gamma, t /\left(\frac{a}{b}\right)\right)\right) \leq 0,
$$

which is also satisfied, since $0<f_{D, X}\left(\gamma, t /\left(\frac{a}{b}\right)\right) \leq f_{N}(\gamma)$.

\section{References}

1. F. J. Arcelus, S. Kumar, G. Srinivasan, The effectiveness of manufacturer vs. retailer rebates within a newsvendor framework, European Journal of Operational Research 219 (2) (2012) 252-263.

2. A. R. Bennato, T. Valletti, Pharmaceutical innovation and parallel trade, International Journal of Industrial Organization 33 (2014) 83-92.

3. D. P. Bertsekas, Nonlinear Programming, Athena Scientific, 2004

4. R. Bishop, Duopoly: Collusion or warfare?, American Economic Review 50 (5) (1960) 933-961.

5. S. Boyd, L. Vandenberghe, Convex Optimization, Cambridge University Press, 2004.

6. K. R. Brekke, A. L. Grasdal, T. H. Holmås, Regulation and pricing of pharmaceuticals: Reference pricing or cap regulation?, European Economics Review 53 (2) (2009) 170-185.

7. X. Chen, C.-L. Li, B.-D. Rhee, D. Simchi-Levi, The impact of manufacturer rebates on supply chain profits, Naval Research Logistics 54 (6) (2007) 667-680.

8. Y. Chen, K. E. Maskus, Vertical price control and parallel imports, Journal of International Trade \& Economic Development 14 (1) (2005) 1-18.

9. P. M. Danzon, The economics of parallel trade, Pharmaeconomics 13 (3) (1998) 293-304.

10. P. M. Danzon, A. Towse, Differential pricing for pharmaceuticals: reconciling access, R \& D and patents, International Journal of Health Care Finance and Economics 3 (3) (2003) 183-205.

11. P. M. Danzon, A. Towse, J. Mestre-Ferrandiz, Value-based differential pricing: efficient pricing for drugs in a global context, Health Economics 24 (3) (2015) 294-301.

12. O. C. Demirag, P. Keskinocak, J. Swann, Customer rebates and retailer incentives in the presence of competition and price discrimination, European Journal of Operational Research 251 (1) (2011) 268-280.

13. R. Duncan Luce, H. Raiffa, Games and Decisions: Introduction and Critical Survey, John Wiley \& Sons, 1957.

14. T. Duso, A. Herr, M. Suppliet, The welfare impact of parallel imports: a structural approach applied to the German market for oral anti-diabetics, Health Economics 23 (9) (2014) 1036-1057.

15. M. Gaggero, G. Gnecco, M. Sanguineti, Approximate dynamic programming for stochastic N-stage optimization with application to optimal consumption under uncertainty, Computational Optimization and Applications 58 (1) (2014) 31-85.

16. M. Ganslandt, K. E. Maskus, Parallel imports and the pricing of pharmaceutical products: Evidence from the European Union, Journal of Health Economics 23 (2004) 1035-1057.

17. R. Gibbons, Game Theory for Applied Economists, Princeton University Press, 1992.

18. H. Gintis, Game Theory Evolving: A Problem-Centered Introduction to Modeling Strategic Interaction, $1^{\text {st }}$ ed., Princeton University Press, 2000.

19. G. Gnecco, M. Sanguineti, Suboptimal solutions to dynamic optimization problems via approximations of the policy functions, Journal of Optimization Theory and Applications 146 (3) (2010) 764-794.

20. S. Guo, B. Hu, H. Zhong, Impact of parallel trade on pharmaceutical firm's profits: rise or fall?, European Journal of Health Economics 14 (2) (2013) 345-35.

21. F. Iravani, S. Dasu, R. Ahmadi, Beyond price mechanisms: How much can service help manage the competition from gray markets?, European Journal of Operational Research 252 (3) (2016) 789-800.

22. I. Jelovac, C. Bordoy, Pricing and welfare implications of parallel imports in the pharmaceutical industry, International Journal of Health Care Finance and Economics 5 (1) (2005) 5-21.

23. P. Kanavos, S. Vandoros, R. Irwin, E. Nicod, M. Casson, Differences in costs of and access to pharmaceutical products in the EU, Directorate-General for Internal Policies, European Parliament (2011).

URL http://www.europarl.europa.eu/RegData/etudes/etudes/join/2011/451481/

24. F. Kermani, Another twist in the EU parallel trade saga?, Pharmaceutical Technology Europe 2 (1) (2010) 14-16.

25. B. Kim, K. S. Park, Organizational structure of a global supply chain in the presence of a gray market: Information asymmetry and valuation difference, International Journal of Production Economics 175 (3) (2016) 71-80. 
26. E. Koutsoupias, C. H. Papadimitriou, Worst-case equilibria, in: Proceedings of the $16^{\text {th }}$ Annual Symposium on Theoretical Aspects of Computer Science, 1999.

27. E. Koutsoupias, C. H. Papadimitriou, Worst-case equilibria, Computer Science Review 3 (2) (2009) 65-69.

28. C. Li, J. Robles, Product innovation and parallel trade, International Journal of Industrial Organization 25 (2006) 417-429.

29. D. G. Luenberger, Optimization by Vector Space Methods, Wiley, 1969.

30. K. E. Maskus, Parallel imports, World Economy 23 (9) (2000) 1269-1284.

31. K. E. Maskus, Y. Chen, Vertical price control and parallel imports: theory and evidence, Review of International Economics 12 (4) (2004) 551-570.

32. K. E. Maskus, M. Ganslandt, Parallel trade in pharmaceutical products: Implications for procuring medicines for poor countries, in: B. Granville (ed.), The Economics of Essential Medicines, Royal Institute of International Affairs, London, 2002, pp. 57-80.

33. F. Müller-Langer, Creating R \& D Incentives for Medicines for Neglected Diseases, Springer Gabler Research, 2009.

34. F. Müller-Langer, Does parallel trade freedom harm consumers in small markets?, Croatian Economic Survey 1 (11) (2009) 11-41.

35. F. Müller-Langer, An analysis of the ambiguous welfare effects of parallel trade freedom, Max Planck Institute for Intellectual Property, Competition \& Tax Law 2010 (2010) 1-29, DOI=http://dx.doi.org/10.2139/ssrn.1535188, Research Paper No. 10-03.

36. F. Müller-Langer, Parallel trade and its ambiguous effects on global welfare, Review of International Economics 20 (1) (2012) 177-185.

37. A. Mutho, Bargaining Theory with Applications, Cambridge University Press, 1999.

38. K. Pauwels, I. Huys, M. Casteels, S. Simoens, Drug shortages in European countries: a trade-off between market attractiveness and cost containment?, BMC Health Services Research 14 (2014) 1-9, article ID=438.

39. F. P. Ramsey, A contribution to the theory of taxation, The Economic Journal 37 (145) (1927) 47-61.

40. U. Religioni, A. Czerw, Economic impact of parallel trade on the selected pharmaceutical markets in the European Union, Progress in Health Sciences 2 (2) (2012) 181-189.

41. R. Schmalensee, Output and welfare implications of monopolistic third-degree price discrimination, The American Economic Review 71 (1) (1981) 242-247.

42. R. Schmalensee, competitive advantage and collusive optima, International Journal of Industrial Organization 5 (4) (1987) $351-368$.

43. J. Sutton, Technology and Market Structure: Theory and History, MIT Press, 1998.

44. J. Tirole, The Theory of Industrial Organization, MIT Press, 1988.

45. A. Towse, M. Pistollato, J. Mestre-Ferrandiz, Z. Khan, S. Kaura, L. Garrison, European Union pharmaceutical markets: A case for differential pricing?, International Journal of the Economics of Business 22 (2) (2015) 263-275.

46. T. M. Valletti, Differential pricing, parallel trade, and the incentive to invest, Journal of International Economics 70 (1) (2006) 314-324.

47. T. M. Valletti, S. Szymanski, Parallel trade, international exhaustion and intellectual property rights: a welfare analysis, Journal of Industrial Economics 54 (4) (2006) 499-526.

48. J. Zhang, The benefits of consumer rebates: A strategy for gray market deterrence, European Journal of Operational Research 251 (2) (2016) 509-521. 